\title{
Entropy and the clique polynomial
}

\section{Citation}

McMullen, C. T. 2014. “Entropy and the Clique Polynomial.” Journal of Topology 8 (1) (November 28): 184-212. doi:10.1112/jtopol/jtu022.

\section{Published Version}

10.1112/jtopol/jtu022

\section{Permanent link}

http://nrs.harvard.edu/urn-3:HUL.InstRepos:24890382

\section{Terms of Use}

This article was downloaded from Harvard University's DASH repository, and is made available under the terms and conditions applicable to Open Access Policy Articles, as set forth at http:// nrs.harvard.edu/urn-3:HUL.InstRepos:dash.current.terms-of-use\#OAP

\section{Share Your Story}

The Harvard community has made this article openly available.

Please share how this access benefits you. Submit a story.

Accessibility 


\title{
Entropy and the clique polynomial
}

\author{
Curtis T. McMullen
}

28 December, 2013

\begin{abstract}
This paper gives a sharp lower bound on the spectral radius $\rho(A)$ of a reciprocal Perron-Frobenius matrix $A \in \mathrm{M}_{2 g}(\mathbb{Z})$, and shows in particular that $\rho(A)^{g} \geq(3+\sqrt{5}) / 2$. This bound supports conjectures on the minimal entropy of pseudo-Anosov maps. The proof is based on a study of the curve complex of a directed graph.
\end{abstract}

\section{Contents}

1 Introduction . . . . . . . . . . . . . . . . . 1

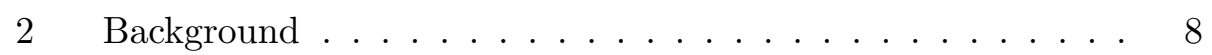

3 The Perron polynomial . . . . . . . . . . . . . . 9

4 The clique polynomial . . . . . . . . . . . . . 14

5 Cones and cohomology . . . . . . . . . . . . . 20

6 Graphs with small growth . . . . . . . . . . . . . 22

7 Reciprocal matrices . . . . . . . . . . . . . . 29

A Appendix: Optimal metrics and outer space . . . . . . . . 34 


\section{Introduction}

In this paper we study the clique polynomial of a graph and its connections to entropy, Perron-Frobenius matrices, pseudo-Anosov maps, right-angled Artin groups and outer space.

These connections provide general methods for analyzing the leading eigenvalue of a non-negative matrix. As a motivating application, we will show:

Theorem 1.1 The minimum value of the spectral radius $\rho(A)$ over all reciprocal Perron-Frobenius matrices $A \in \mathrm{M}_{2 g}(\mathbb{Z}), g \geq 2$, is given by the largest root of the polynomial

$$
L_{2 g}(t)=t^{2 g}-t^{g}\left(1+t+t^{-1}\right)+1 .
$$

Consequently $\rho(A)^{g} \geq(3+\sqrt{5}) / 2$ for all such $A$.

Here reciprocal means the eigenvalues of $A$ (counted with multiplicities) are invariant under $\mu \mapsto \mu^{-1}$. This hypothesis is of interest for applications to the mapping class group (see below).

The Perron polynomial. Let $(\Gamma, m)$ be a finite, metrized, directed graph. The metric is given by a positive function $m: E(\Gamma) \rightarrow \mathbb{R}_{+}$, specifying the length of each edge; we allow parallel edges and loops. We define the Perron polynomial of $(\Gamma, m)$ by

$$
P(t)=\operatorname{det}(I-A(t)),
$$

where $A(t)$ is the weighted adjacency matrix with entries

$$
A_{a b}(t)=\sum_{[e]=(a, b)} t^{m(e)}
$$

Here $[e]=(a, b)$ if the edge $e$ runs from vertex $a$ to vertex $b$. The Perron polynomial is a finite sum of the form $P(t)=\sum a_{\alpha} t^{\alpha}$, with integral coefficients and real exponents $\alpha \geq 0$.

The growth rate of a metrized directed graph is defined by

$$
\lambda(\Gamma, m)=\lim _{T \rightarrow \infty} N_{0}(T)^{1 / T},
$$

where $N_{0}(T)$ is the number of closed directed paths in $\Gamma$ of length $\leq T$. It is related to the Perron polynomial by the following basic result ( $\S 3)$ : 
Theorem 1.2 The smallest positive zero of $P(t)$ is given by $t=1 / \lambda(\Gamma, m)$. The quantity $h(m)=\log \lambda(\Gamma, m)$ is a convex function of $m$.

Eigenvalues. The growth rate $\lambda(\Gamma, 1)$, obtained by setting $m(e)=1$ for all $e$, is the spectral radius of the traditional adjacency matrix $A_{\Gamma}=A(1)$. When $m$ takes integer values, we can put $m(e)-1$ new vertices along each edge $e \in E(\Gamma)$ to obtain a new graph $\Delta$; then

$$
\rho\left(A_{\Delta}\right)=\lambda(\Delta, 1)=\lambda(\Gamma, m) .
$$

Thus $P(t)$ packages information on the eigenvalues of infinitely many directed graphs with the same underlying topology.

The clique polynomial. Now let $G$ be a finite, undirected graph, with no loops or parallel edges, and let $w: V(G) \rightarrow \mathbb{R}_{+}$be a weight on the vertices of $G$. The vertices $K \subset V(G)$ form a clique if they span a complete subgraph; we allow $K=\emptyset$. The clique polynomial of $(G, w)$ is defined by

$$
Q(t)=\sum_{K}(-1)^{|K|} t^{w(K)},
$$

where $w(K)=\sum_{v \in K} w(v)$ and the sum is over all cliques of $G$.

The graph $G$ also determines a right-angled Artin group $W(G)$. The group $W(G)$ is obtained from the free group on the vertices $V(G)$ by imposing the relation $[a, b]=1$ whenever $a$ and $b$ are joined by an edge. The weight of an element $g=a_{1} \cdots a_{n}$ in the positive semigroup $W(G)_{+}$is defined by

$$
w(g)=\sum w\left(a_{i}\right)
$$

it is independent of the word chosen to express $g$. The growth rate of $(G, w)$ is defined by

$$
\lambda(G, w)=\lim _{T \rightarrow \infty} N(T)^{1 / T},
$$

where $N(T)=\left|\left\{g \in W(G)_{+}: 0<w(g) \leq T\right\}\right|$.

Given an ordering of $V(G)$, the lexicographically minimal words in $W(G)_{+}$ are recognized by a finite-state automaton. Allowed transitions between states are described by a directed graph $\Delta$. Using this fact, Theorem 1.2 and results from $[\mathrm{CF}]$, in $\S 4$ we will show:

Theorem 1.3 The smallest positive zero of $Q(t)$ is given by $t=1 / \lambda(G, w)$, and the function

$$
h(w)=\log \lambda(G, w)
$$

is convex. Provided $G^{\prime}$ is connected and $|V(G)|>1$, the function $h(w)$ is strictly convex, real-analytic, and $h(w) \rightarrow \infty$ at the boundary of the cone of positive weights. 
Here $G^{\prime}$ denotes the graph with the same vertices as $G$ but the complementary edges. When $G^{\prime}$ has $k$ components, we have a factorization of the form

$$
W(G) \cong W\left(G_{1}\right) \times W\left(G_{2}\right) \cdots \times W\left(G_{k}\right) .
$$

The condition that $G^{\prime}$ is connected ensures that $W(G)$ is irreducible, in the sense that $k=1$.

The curve complex. The polynomials $P(t)$ and $Q(t)$ are related by the curve complex construction.

A simple curve is a collection of edges $C \subset E(\Gamma)$ that form a closed, directed loop which never visits the same vertex twice. A multicurve is a union of simple curves $C=C_{1} \cup \cdots \cup C_{k}$ such that no two share a vertex.

The curve complex of $\Gamma$ is the graph $G$ obtained by taking a vertex for each simple curve $C$, and then joining $C_{1}$ and $C_{2}$ by an edge whenever $\left(C_{1}, C_{2}\right)$ is a multicurve. The terminology is meant to recall the curve complex of a surface [Ha], [MM], although for our purposes the 1-skeleton $G$ will suffice.

The metric $m$ on $\Gamma$ determines weights $w$ on $G$ by

$$
w(C)=\sum_{e \in C} m(e)
$$

By a basic result in graph theory [CDS, §1.4], we have:

Theorem 1.4 The Perron polynomial of $(\Gamma, m)$ is equal to the clique polynomial of its curve complex $(G, w)$. That is,

$$
P(t)=Q(t)=\sum_{C}(-1)^{k(C)} t^{m(C)},
$$

where the sum is over all multicurves in $\Gamma$. In particular we have

$$
\lambda(\Gamma, m)=\lambda(G, w),
$$

where $w$ is given by (1.2).

Here $k(C)$ denotes the number of components of $C$. (For the proof, express $\operatorname{det}(I-A(t))$ as a sum over permutations of $V(\Gamma)$ and write each permutation as a product of disjoint cycles.)

This shows Theorem 1.3 implies Theorem 1.2 and is formally stronger, since some graphs (e.g. $G=2 A_{2}$ ) do not occur as curve complexes. 


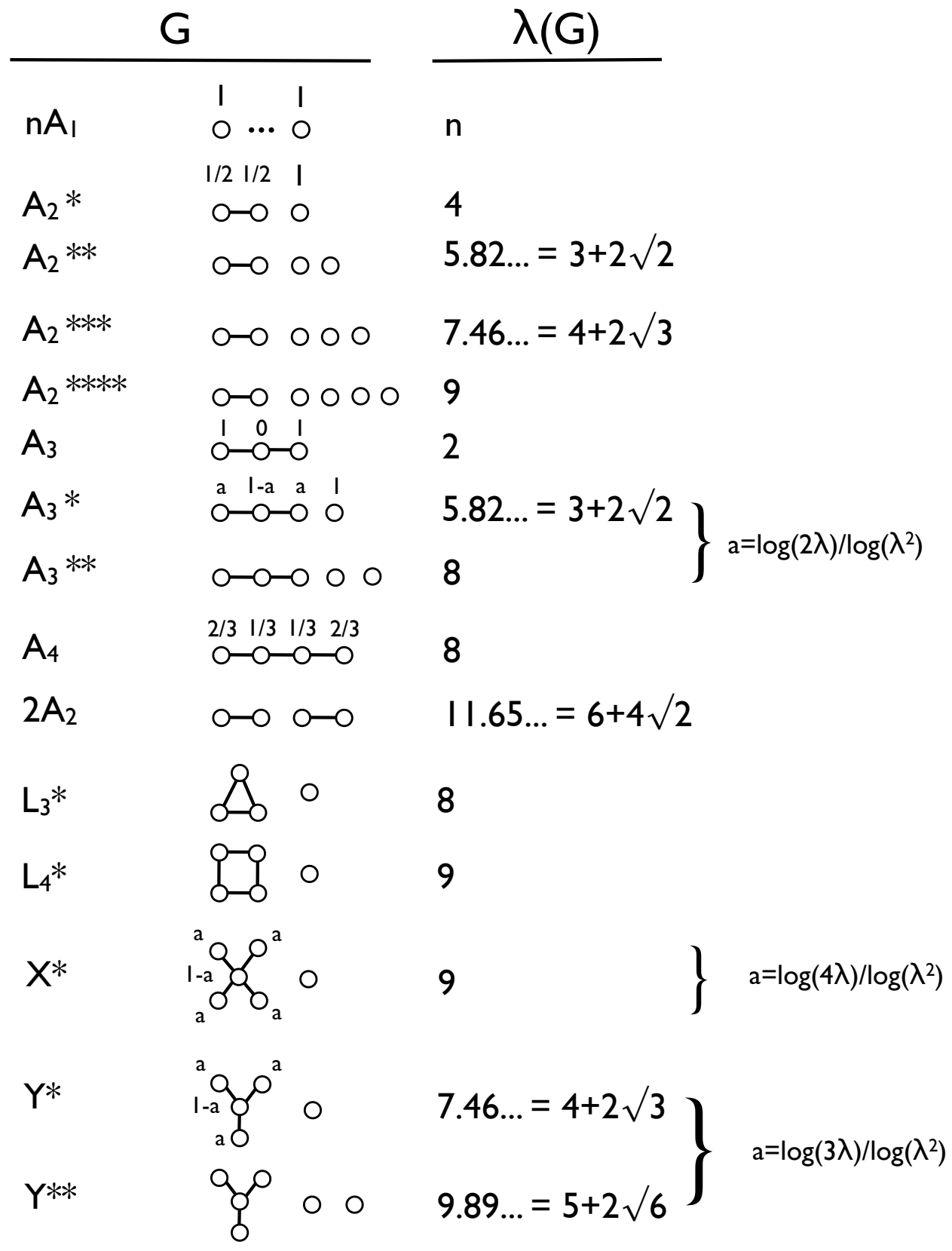

Figure 1. Minimal growth rates $\lambda(G)$. 
Connectivity. A directed graph $\Gamma$ with curve complex $G$ is strongly connected if every pair of vertices lies on a closed, directed path. In this case $G^{\prime}$ is connected. This hypothesis will occur frequently below.

Classification. A weight $w: V(G) \rightarrow \mathbb{R}_{+}$is admissible if $w(K) \leq 1$ for all cliques $K \subset V(G)$. We define the minimal growth rate of $G$ by

$$
\lambda(G)=\inf \{\lambda(G, w): w \text { is an admissible weight }\} .
$$

Similarly, a metric on $\Gamma$ is admissible if $m(C) \leq 1$ for all multicurves $C$, and we let

$$
\lambda(\Gamma)=\inf \{\lambda(\Gamma, m): m \text { is an admissible metric }\} .
$$

Any metric of total length 1 is admissible, as is the metric $m(e)=1 / n$ where $n=|V(\Gamma)|$. It is thus immediate that we have:

$$
\lambda(\Gamma, 1)^{n} \geq \lambda(\Gamma) \geq \lambda(G)
$$

when $G$ is the curve complex of $\Gamma$.

In $\S 6$ we will show:

Theorem 1.5 For any $M>0$, there are only finitely many graphs $G$ such that $G^{\prime}$ is connected and $1<\lambda(G) \leq M$.

Theorem 1.6 The graphs with $G^{\prime}$ connected and $1<\lambda(G)<8$ are given by

$$
A_{2}^{*}, A_{2}^{* *}, A_{2}^{* * *}, A_{3}^{*}, Y^{*} \text { and } n A_{1},
$$

with $2 \leq n \leq 7$.

These graphs, along with some borderline cases, are shown in Figure 1. This result can be regarded as a classification of right-angled Artin semigroups with small growth. See $\S 4$ for notation and $\S 6$ for more details.

Perron-Frobenius matrices. There are thousands of strongly connected, trivalent directed graphs (up to isomorphism) with $1<\lambda(\Gamma)<8$. But these graphs give rise to only a handful of curve complexes, by Theorem 1.6 (see Table 2 and Figure 3). In $\S 7$ we leverage this combinatorial simplification, together with equation (1.3), to establish the bound on $\rho(A)$ given in Theorem 1.1. For general Perron-Frobenius matrices of rank $n \geq 2$, we will show that the minimum of $\rho(A)$ is the largest root of $t^{n}-t-1=0$. For irreducible $A$ with $\rho(A)>1$ one has $\rho(A)^{n} \geq 2$ [Pen, p. 445]; see also [HS, Lemma 1.3]. 


\begin{tabular}{|c||c|c|c|c|c|c|c|c|c|c|c|}
\hline$G$ & $2 A_{1}$ & $3 A_{1}$ & $4 A_{1}$ & $5 A_{1}$ & $6 A_{1}$ & $7 A_{1}$ & $A_{2}^{*}$ & $A_{2}^{* *}$ & $A_{2}^{* * *}$ & $A_{3}^{*}$ & $Y^{*}$ \\
\hline$\# \Gamma$ & 1 & 2 & 14 & 119 & 1556 & 26,286 & 1 & 5 & 42 & 5 & 42 \\
\hline
\end{tabular}

Table 2. Number of trivalent $\Gamma$ with a given curve complex $G$.
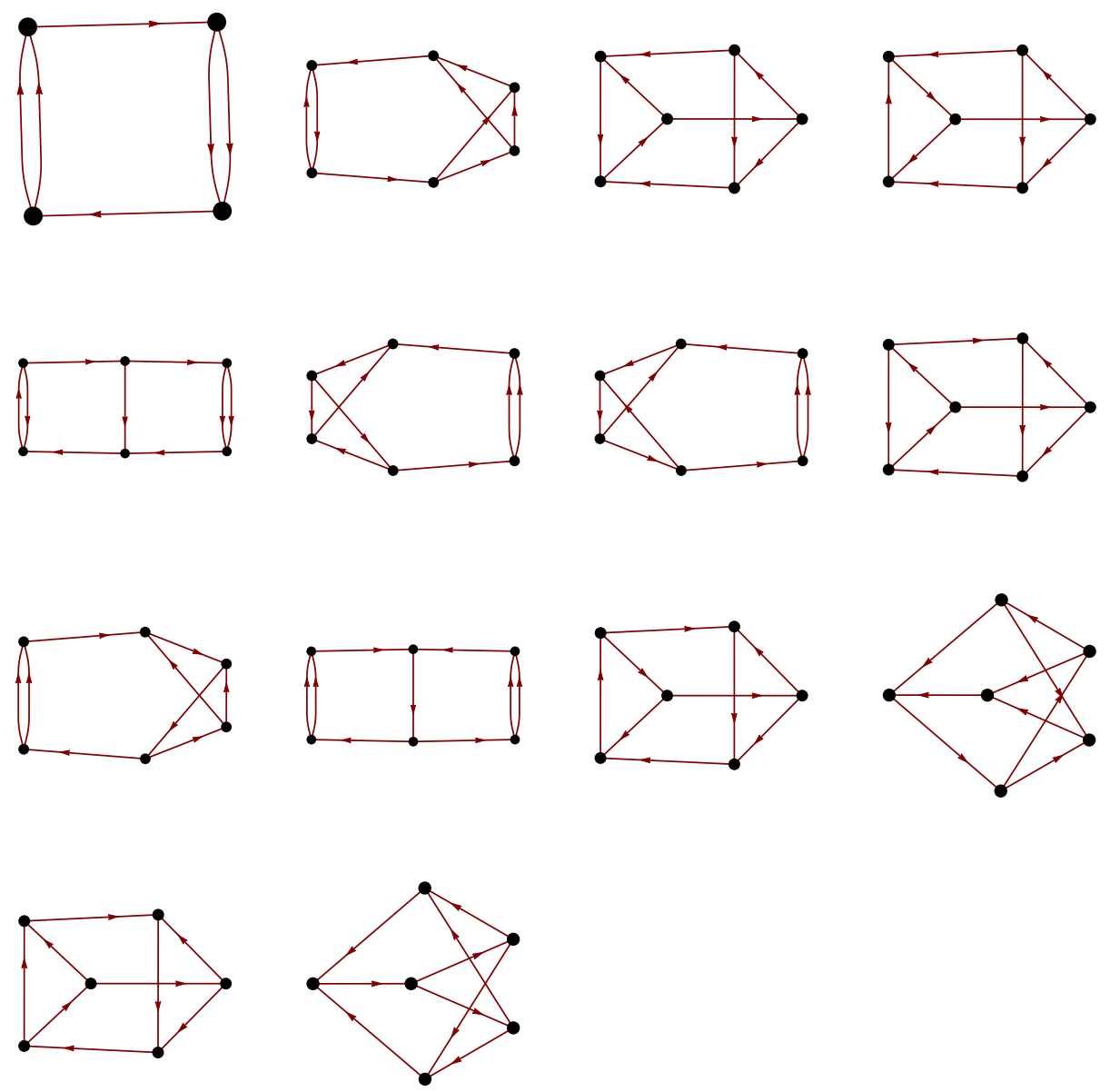

Figure 3. Example: The trivalent graphs $\Gamma$ with $G=4 A_{1}$. 
Pseudo-Anosov maps. The formulation of Theorem 1.1 is motivated by questions in surface dynamics raised by Lanneau-Thiffeault and Hironaka [LT, Question 6.1], [Hir, Question 1.12].

Let $\Sigma_{g}$ be a closed surface of genus $g$, and let $\log \delta_{g}$ denote the minimum of the entropy $h(f)$ over all pseudo-Anosov mapping-classes $f \in \operatorname{Mod}\left(\Sigma_{g}\right)$. Let $\delta_{g}^{+}$denote the minimum for $f$ with orientable foliations. LanneauThiffeault asked if, for $g$ even, $\delta_{g}^{+}$coincides with the largest zero of the polynomial $L_{2 g}(t)$, and proved results in this direction for $g \leq 8$. $^{1}$

The question of convergence of $\delta_{g}^{g}$ was raised in [Mc, $\left.\S 10\right]$. Hironaka showed $\lim \sup \delta_{g}^{g} \leq(3+\sqrt{5}) / 2$ and asked if, in fact,

$$
\lim _{g \rightarrow \infty} \delta_{g}^{g}=\lim \left(\delta_{g}^{+}\right)^{g}=\frac{(3+\sqrt{5})}{2} .
$$

For a positive answer, one needs a lower bound on $\delta_{g}^{g}$.

A key feature of a surface automorphism $f$ is that it preserves the symplectic form on $H_{1}\left(\Sigma_{g}\right)$ and, more generally, on the tangent space to $\mathcal{M} \mathcal{L}_{g}$ (see e.g. [Pa]). Thus a suitable train-track representative for $f$ gives rise to a Perron-Frobenius matrix $A$ with $h(f)=\log \rho(A)$, whose eigenvalues come in reciprocal pairs. Theorem 1.1 may therefore be useful for bounding $h(f)$ from below, if the complexity of the train track - and hence the rank of $A$ - can be controlled.

The Perron polynomial of a directed graph enjoys many similarities with the Teichmüller polynomial of a fibered 3-manifold, which plays a useful role in the construction of mapping-classes with small entropy ( $\$ 5)$. In $\S 6$ we give examples of the transcendence of optimal weights, a phenomena which was first observed in the setting of fibered 3-manifolds [Sun].

Optimal metrics and outer space. In the Appendix we discuss optimal metrics on undirected graphs, and the connection with entropy as a piecewisesmooth Morse function on Culler-Vogtmann's outer space $X_{n}$.

Symplectic groups. For comparison, we remark that it is an open problem to show that $\lim \inf \eta_{g}^{g}>0$, where

$$
\eta_{g}=\inf \left\{\rho(A): A \in \operatorname{Sp}_{2 g}(\mathbb{Z}), \rho(A)>1\right\} .
$$

This is equivalent to a conjecture of Schinzel and Zassenhaus from 1965 (as is same statement for $\mathrm{GL}_{g}(\mathbb{Z})$ ). For recent results, see e.g. [Smy, §3].

Notes and references. The convexity of $h(m)$ can be regarded as an instance of the convexity of pressure in the thermodynamic formalism [PP].

\footnotetext{
${ }^{1}$ See also $[\mathrm{CH}]$ for the case $g=2$, and [Hir], [AD] and [KT2] for further progress on the values of $\delta_{g}^{+}$.
} 
Some useful references for graph theory and the clique polynomial include $[\mathrm{CDS}],[\mathrm{CF}],[\mathrm{Fi}],[\mathrm{Csi}]$, and [GS]. For more on the related algebraic theory of Perron numbers, see e.g. [Li], [B], [Smy], [KOR] and [Wu]. We learned of the relevance of [CDS, §1.4] and Theorem 1.4 from Birman's preprint [Bir].

I would like to thank E. Hironaka for many useful discussions related to this work, which began during her visit to Harvard in 2009; and H. Sun for helpful comments.

\section{Background}

In this section we recall some basic facts about positive power series and positive matrices.

Polynomials and power series. In the sequel it will be useful to count paths, cycles, group elements and other objects using formal generating functions. The functions we will consider have the form

$$
f(t)=a_{0}+\sum_{n=1}^{\infty} a_{n} t^{\alpha_{n}}
$$

with $a_{n} \in \mathbb{Z}, \alpha_{n} \in \mathbb{R}$, and $0<\alpha_{n} \rightarrow \infty$ as $n \rightarrow \infty$. The ring of all such formal power series will be denoted by $\mathbb{Z}\left[\left[t^{\alpha}: \alpha>0\right]\right]$. The subring of finite sums (polynomials with positive real exponents) will be denoted by $\mathbb{Z}\left[t^{\alpha}: \alpha>0\right]$.

Note that $t \mapsto t^{\alpha}$ has a unique analytic continuation from the positive real axis to the slit complex plane $U=\mathbb{C} \backslash(-\infty, 0]$. If $f(t)$ is a finite sum, then it defines an analytic function on the whole region $U$. Otherwise, we have limsup $\left|a_{n}\right| \geq 1$ and hence the sum (2.1) defining $f(t)$ diverges for $|t|>1$. To determine its region of convergence, let

$$
\lambda=\limsup _{T \rightarrow \infty} N(T)^{1 / T} \quad \text { where } \quad N(T)=\sum\left\{\left|a_{i}\right|: \alpha_{i} \leq T\right\},
$$

and let $\tau=1 / \lambda$. Then the sum (2.1) is absolutely convergent for $|t|<\tau$, and defines an analytic function on the region $U \cap B(0, \tau)$. Moreover, if $a_{n} \geq 0$ for all $n$, then:

1. The series $\sum a_{n} t^{\alpha_{n}}$ diverges for $t>\tau$, and

2. The analytic function $f(t)$ has a singularity at $t=\tau$. 
Indeed, the substitution $t=e^{-s}$ converts (2.1) into a traditional Dirichlet series of the form

$$
F(s)=\sum_{0}^{\infty} a_{n} e^{-\alpha_{n} s},
$$

and the statements above follow well-known results on the abscissa of convergence and boundary behavior of $F(s)$; see [La, §243] and [HR, Thms. 8 and 10].

Perron-Frobenius theory. Let $A \in \mathrm{M}_{n}(\mathbb{R})$ be a real matrix of rank $n$. We will write $A \geq 0$ (respectively $A \gg 0$ ) to mean that $A_{i j} \geq 0$ (respectively $\left.A_{i j}>0\right)$ for all indices $1 \leq i, j \leq n$, and similarly for vectors $v=\left(v_{i}\right) \in \mathbb{R}^{n}$. The notation $A \geq B$ means $A-B \geq 0$.

There are many equivalent norms on the space of matrices; for concreteness, let

$$
\|A\|=\sum_{i, j}\left|A_{i j}\right| .
$$

The spectral radius $\rho(A)$, defined as the maximum modulus of the complex eigenvalues of $A$, satisfies

$$
\rho(A)=\lim _{n \rightarrow \infty}\left\|A^{n}\right\|^{1 / n} .
$$

If $A \geq 0$ then $\rho(A)$ is in fact an eigenvalue of $A$, with an associated eigenvector $v \geq 0$, and

$$
A \geq B \geq 0 \Longrightarrow \rho(A) \geq \rho(B) \geq 0 .
$$

A matrix is Perron-Frobenius if $A^{m} \gg 0$ for some $m>0$. In this case $\rho(A)$ is a simple eigenvalue for $A$, it has an associated eigenvector $v \gg 0$, and the remaining eigenvalues of $A$ satisfy $|\lambda|<\rho(A)$.

A matrix $A \geq 0$ is irreducible if $B=A+A^{2}+\cdots+A^{m} \gg 0$ for some $m>$ 0 . In this case $\rho(A)$ is still a simple eigenvalue for $A$, and its corresponding eigenvector is strictly positive, but $A$ may have other eigenvalues satisfying $|\lambda|=\rho(A)$. For these facts, see e.g. [Gant, Ch. XIII].

Note that if $A$ is the adjacency matrix of a graph $\Gamma$, then $A$ is irreducible iff $\Gamma$ is strongly connected.

\section{The Perron polynomial}

In this section we record some basic facts about directed graphs, including the proof of Theorem 1.2. 
Directed graphs. Let $\Gamma$ denote a finite directed graph as in $\S 1$. The graph $\Gamma$ is determined by its edge and vertex sets together with the map

$$
E(\Gamma) \rightarrow V(\Gamma) \times V(\Gamma)
$$

which sends an edge $e$ running from $a$ to $b$ to the ordered pair $[e]=(a, b)$. We allow $[e]=(a, a)$ and we may have $\left[e_{1}\right]=\left[e_{2}\right]$ even if $e_{1} \neq e_{2}$. Alternatively, if we regard $\Gamma$ as a topological 1-complex, then the same information is recorded by the boundary map

$$
\partial: C_{1}(\Gamma) \cong \mathbb{Z}^{E(\Gamma)} \rightarrow C_{0}(\Gamma) \cong \mathbb{Z}^{V(\Gamma)},
$$

which is given by $\partial(e)=b-a$ if $[e]=(a, b)$.

We let $H_{1}(\Gamma)=Z_{1}(\Gamma)=\operatorname{Ker}(\partial)$ denote the space of integral 1-cycles on $\Gamma$.

Paths and simple curves. A path is a sequence of edges $p=\left(e_{1}, \ldots, e_{n}\right)$, $n \geq 1$, with $\left[e_{i}\right]=\left(a_{i}, b_{i}\right)$ and $a_{i+1}=b_{i}$; it is closed if $a_{1}=b_{n}$. A closed path that never visits the same vertex twice determines a simple curve

$$
C=\sum_{1}^{n} e_{i} \in H_{1}(\Gamma) .
$$

A pair of simple curves are disjoint if they have no vertices in common. A multicurve is a sum of disjoint simple curves, $C=C_{1}+\cdots+C_{k}$.

The Perron polynomial. From $\Gamma$ we obtain an adjacency matrix $A_{\Gamma}$ with entries in the group ring $\mathbb{Z}\left[C_{1}(\Gamma)\right]$, defined by

$$
\left(A_{\Gamma}\right)_{a b}=\sum_{[e]=(a, b)} e .
$$

The Perron polynomial of $\Gamma$ is defined by $P_{\Gamma}=\operatorname{det}\left(I-A_{\Gamma}\right)$. By Theorem 1.4 we have

$$
P_{\Gamma}=\sum_{C}(-1)^{k(C)} \cdot C \in \mathbb{Z}\left[H_{1}(\Gamma)\right]
$$

where the sum is over all multicurves and $k(C)$ denotes the number of components of $C$. (We include $C=0$ with $k(C)=0$.)

Metrics. A metric on $\Gamma$ is a map $m: E(\Gamma) \rightarrow \mathbb{R}$ such that $m(e)>0$ for all $e$. Since $E(\Gamma)$ gives a basis for $C_{1}(\Gamma)$, the map $e \mapsto t^{m(e)}$ extends to a ring homomorphism

$$
\mathbb{Z}\left[C_{1}(\Gamma)\right] \rightarrow \mathbb{Z}\left[t^{\alpha}: \alpha>0\right]
$$


sending $e$ to $t^{m(e)}$. The image of $P_{\Gamma}=\sum a_{C} \cdot C$ under this map, given by

$$
P_{\Gamma}\left(t^{m}\right)=\sum_{C} a_{C} t^{m(C)}
$$

is the Perron polynomial of $(\Gamma, m)$. Equivalently, $m$ determines a matrix $A_{\Gamma}\left(t^{m}\right)$ whose coefficients lie in $\mathbb{Z}\left[t^{\alpha}: \alpha>0\right]$, and we have

$$
P_{\Gamma}\left(t^{m}\right)=\operatorname{det}\left(I-A_{\Gamma}\left(t^{m}\right)\right)
$$

As in $\S 1$, we often write $A(t)=A_{\Gamma}\left(t^{m}\right)$ and $P(t)=P_{\Gamma}\left(t^{m}\right)$ when the metric is fixed, and think of $A(t)$ and $P(t)$ as real-analytic functions defined for all $t \geq 0$.

Growth rates. Let $N(T)$ denote the number of paths $p=\left(e_{1}, \ldots, e_{n}\right)$ in $\Gamma$ with length

$$
m(p)=\sum_{1}^{n} m\left(e_{i}\right) \leq T,
$$

and let $N_{0}(T)$ be the number of closed paths satisfying the same inequality.

Lemma 3.1 The growth rate of closed paths,

$$
\lambda(\Gamma, m)=\lim _{T \rightarrow \infty} N_{0}(T)^{1 / T},
$$

is well-defined for all metrized directed graphs. It agrees with the growth rate of all paths, provided $\Gamma$ has at least one cycle.

Proof. Any path $p$ of length $\leq T_{1}+T_{2}$ can be split into segments $p_{1}, p_{2}$ and $r$ with $m\left(p_{1}\right) \leq T_{1}, m\left(p_{2}\right) \leq T_{2}$ and $m(r)=O(1)$. Thus $N(T)$ is roughly submultiplicative: it satisfies the inequality

$$
N\left(T_{1}+T_{2}\right) \leq K \cdot N\left(T_{1}\right) N\left(T_{2}\right),
$$

and hence $N(T)^{1 / T}$ converges to a finite limit.

Now if $\Gamma$ is strongly connected, any path can be continued a bounded distance to form a cycle; thus $N_{0}(T) \asymp N(T)$ and therefore $N_{0}(T)^{1 / T}$ and $N(T)^{1 / T}$ have the same limit. The general case is handled by considering the strongly connected components of $\Gamma$. 
Extreme case. If $\Gamma$ has no closed paths, then $A_{\Gamma}$ is nilpotent, $P_{\Gamma}=1$ and $\lambda(\Gamma, m)=0$ for all $m$.

The matrices $A(t)$ associated to a metrized graph $(\Gamma, m)$ are increasing with $t$. More precisely, let $\mu=\min _{e \in E(\Gamma)} m(e)>0$ be the length of the shortest edge in $\Gamma$; then we have

$$
t \frac{d}{d t} A(t) \geq \mu \cdot A(t)
$$

which implies

$$
t \frac{d}{d t} \rho(A(t)) \geq \mu \cdot \rho(A(t))
$$

for all $t \geq 0$ by $(2.2)$.

Trace formula. Let $\mathcal{C}$ denote the set of all closed paths in $\Gamma$. By Cramer's rule, there is a matrix of polynomials $B(t)$ (built from minors of $A(t)$ ) such that

$$
B(t)(I-A(t))=\operatorname{det}(I-A(t)) I=P(t) I .
$$

Taking traces, we obtain a formal expression for the generating function of the lengths of closed paths:

$$
f(t)=\sum_{p \in \mathcal{C}} t^{m(p)}=\sum_{n=1}^{\infty} \operatorname{Tr} A^{n}(t)=\operatorname{Tr} \frac{A(t)}{I-A(t)}=\frac{R(t)}{P(t)}
$$

where $R(t)=\operatorname{Tr}(A(t) B(t)) \in \mathbb{Z}\left[t^{\alpha}: \alpha>0\right]$.

Theorem 3.2 If $\Gamma$ has at least one closed path, then the smallest positive zero of $P(t)$ is given by $\tau=1 / \lambda(\Gamma, m)$. This is also the unique positive solution to the equation $\rho(A(t))=1$.

Proof. By general results $(\S 2)$, the generating function $f(t)$ given in equation (3.4) has radius of convergence $\tau$ given by the reciprocal of the growth rate $\lambda(\Gamma, m)$. Moreover $f(t)$ has a singularity at $t=\tau$, so $\tau$ must be among the zeros of $P(t)$. Since $\Gamma$ has at least one cycle, $\rho(A(t))$ is positive for $t>0$. Equation (3.3) then implies $\rho(A(t))$ is strictly increasing and tends to infinity with $t$, so there is a unique $t_{0}>0$ such that $\rho\left(A\left(t_{0}\right)\right)=1$. Since the trace is a sum of eigenvalues, $\sum \operatorname{Tr} A^{n}(t)$ converges for $t<t_{0}$ and diverges for $t>t_{0}$; thus $t_{0}=\tau$. Finally $P(t)=\operatorname{det}(I-A(t)) \neq 0$ for $0<t<\tau$, since in this range $\rho(A(t))<1$, so $\tau$ is in fact the smallest positive zero of $P(t)$. 
Convexity. We now turn to properties of the map $m \mapsto \lambda(\Gamma, m)$.

Theorem 3.3 The quantity $h(m)=\log \lambda(\Gamma, m)$ is a convex function of $m$.

Proof. Any map $s: E(\Gamma) \rightarrow \mathbb{R}$ determines a real-valued matrix $A(\exp (s))$ with entries

$$
A_{a b}(\exp (s))=\sum_{[e]=(a, b)} \exp (s(e)) .
$$

By differentiating it is easy to check that

$$
F_{n}(s)=\frac{1}{n} \log \left(\sum_{a, b} A_{a b}^{n}(\exp (s))\right)
$$

defines a convex, increasing function of $s$ (cf. [Mc, Appendix]), and hence

$$
F(s)=\lim F_{n}(s)=\log \rho(A(\exp (s)))
$$

is convex as well. As we have just seen, the value of $h(m)$ can be characterized by the relation

$$
F(-h(m) m)=0
$$

Now suppose $F\left(-h_{1} m_{1}\right)=F\left(-h_{2} m_{2}\right)=0$. Then by convexity,

$$
s=\frac{h_{2}\left(h_{1} m_{1}\right)+h_{1}\left(h_{2} m_{2}\right)}{h_{1}+h_{2}}=\frac{2 h_{1} h_{2}}{h_{1}+h_{2}} \cdot \frac{\left(m_{1}+m_{2}\right)}{2}
$$

satisfies $F(-s) \leq 0$. This implies the inequality

$$
h\left(\frac{m_{1}+m_{2}}{2}\right) \leq \frac{2 h_{1} h_{2}}{h_{1}+h_{2}} \leq \frac{\left(h_{1}+h_{2}\right)^{2}}{2\left(h_{1}+h_{2}\right)}=\frac{h_{1}+h_{2}}{2},
$$

which gives convexity.

Theorem 3.4 If $\Gamma$ is strongly connected, then $h(m)$ is a real-analytic function of $m$.

Proof. In this case the leading eigenvalue $\rho(A(t))$ of $A(t)$ has multiplicity one, and hence it varies real-analytically with $t$ and $m$. Since the relation $\rho(A(t))=1$ determines $h(m)$, the result follows from the implicit function theorem, using equation (3.2) to verify transversality. 


\section{The clique polynomial}

In this section we relate the clique polynomial of a weighted graph $(G, w)$ to the growth rate $\lambda(G, w)$ of the associated right-angled Artin semigroup $W(G)_{+}$. To do so we construct a directed graph $\Delta$ and a projection $\pi$ : $E(\Delta) \rightarrow V(G)$ such that

$$
\lambda(G, w)=\lambda(\Delta, w \circ \pi)
$$

for any weight $w$. Basic convexity properties of $\lambda(G, w)$, stated in Theorem 1.3 , then follow from the results of $\S 3$. When $G^{\prime}$ is connected, they can be sharpened as follows:

Theorem 4.1 Assume $G^{\prime}$ is connected and $|V(G)|>1$. Then the function

$$
h(w)=\log \lambda(G, w)
$$

is real-analytic, strictly convex, and $h(w) \rightarrow \infty$ at the boundary of the cone of positive weights.

Notation for graphs. Let $G$ be a finite undirected graph, as in $\S 1$. The graph $G$ is determined by a set of vertices $V(G)$ and a set of edges

$$
E(G) \subset \mathcal{P}_{2}(V(G))=\{\{a, b\} \subset V(G): a \neq b\} .
$$

Our notation for graphs is illustrated in Figure 1. We let $A_{n}$ and $L_{n}$ denote an interval and a loop with $n$ vertices. The complete graph on $n$ vertices is denoted $K_{n}$. We write $G=G_{1}+G_{2}$ for the disjoint union of two graphs, and $k G$ for the union of $k$ disjoint copies of $G$. The abbreviations $G^{*}, G^{* *}, \ldots$ denote $G+A_{1}, G+2 A_{1}$, etc.

The join of two graphs, $G * H$, is obtained from $G+H$ by adding all possible edges between $G$ and $H$. The complete bipartite graph $K_{p, q}$ is given by the join $\left(p A_{1}\right) *\left(q A_{1}\right)$. We use the shorthand $X$ and $Y$ for $K_{1,4}$ and $K_{1,3}$ respectively.

The complement $G^{\prime}$ of $G$ is defined by $V(G)=V\left(G^{\prime}\right)$ and $E\left(G^{\prime}\right)=$ $\mathcal{P}_{2}(V(G))-E(G)$. Any graph factors canonically as a join $G=G_{1} * \cdots * G_{n}$, where $G_{1}^{\prime}, \ldots, G_{n}^{\prime}$ are the connected components of $G^{\prime}$. A graph with $G^{\prime}$ connected is 'prime', in the sense that it cannot be written as a nontrivial join $G_{1} * G_{2}$.

A subset $A \subset V(G)$ determines an induced subgraph with vertices $A$, which contains all the edges of $G$ with endpoints in $A$. 
The clique polynomial. The vertices $K \subset V(G)$ form a clique if every pair of distinct vertices in $K$ is joined by edge. The empty set is always a clique.

We may identify $K$ with the 0 -chain

$$
[K]=\sum_{v \in K} v \in C_{0}(G) \cong \mathbb{Z}^{V(G)} .
$$

The clique polynomial of $G$ is defined by

$$
Q_{G}=\sum_{K}(-1)^{|K|}[K] \in \mathbb{Z}\left[C_{0}(G)\right]
$$

The clique polynomial of the weighted graph $(G, w)$ is defined by

$$
Q_{G}\left(t^{w}\right)=\sum_{K}(-1)^{|K|} t^{w(K)} \in \mathbb{Z}\left[t^{\alpha}: \alpha>0\right] .
$$

Here $w: V(G) \rightarrow \mathbb{R}_{+}$and $w(K)=\sum_{a \in K} w(a)$. We write $Q(t)=Q_{G}\left(t^{w}\right)$ when $(G, w)$ is fixed.

Sums and products. It is routine to verify that, consistent with our notation for graphs, the clique polynomial satisfies:

$$
Q_{G+H}=Q_{G}+Q_{H}-1 \text { and } Q_{G * H}=Q_{G} \cdot Q_{H} .
$$

Right-angled Artin groups. A graph $G$ with $V(G)=\left\{a_{1}, \ldots, a_{n}\right\}$ naturally determines a right-angled Artin group, with the presentation

$$
\left.W(G)=\left\langle a_{1}, \ldots, a_{n}\right|\left[a_{i}, a_{j}\right]=1 \text { if }\left(a_{i}, a_{j}\right) \in E(G)\right\rangle .
$$

Let $W(G)_{+} \subset W(G)$ denote the positive semigroup (with identity) on these generators. Every $g \in W(G)_{+}$can be represented by one or more words $\widetilde{g}=v_{1} \cdots v_{k}$ in the free semigroup generated by $V(G)$. In this case we write $g=[\widetilde{g}]$. The number of occurrences of $a_{i}$ in $\widetilde{g}$ depends only on $g$, as can be seen by abelianizing $W(G)$; thus the weight

$$
w(g)=w\left(v_{1} \cdots v_{k}\right)=\sum_{1}^{k} w\left(v_{i}\right)
$$

is well-defined. In fact $[\widetilde{g}]=[\widetilde{h}]$ if and only if $\widetilde{g}$ can be obtained from $\widetilde{h}$ by repeatedly swapping adjacent, commuting letters. 
The generating function. By [CF, Intro. (2) and Thm. 2.4], the reciprocal of the clique polynomial gives the formal generating function for the weights of elements of $W(G)_{+}$: we have

$$
f(t)=\sum_{g \in W(G)_{+}} t^{w(g)}=\frac{1}{Q(t)} .
$$

An automaton for minimal words. Recall that the growth rate of $(G, w)$ is defined by

$$
\lambda(G, w)=\lim _{T \rightarrow \infty}\left|\left\{g \in W(G)_{+}: 0<w(g)<T\right\}\right|^{1 / T} .
$$

We wish to relate the growth of $G$ to the growth of paths in a directed graph $(\Delta, m)$.

To construct $\Delta$, first choose an ordering for the elements of $V(G)$. The corresponding dictionary ordering of the words of length $k$ in $W(G)_{+}$is defined by

$$
v_{1} \cdots v_{k}<v_{1}^{\prime} \cdots v_{k}^{\prime}
$$

if the first two letters that fail to match satisfy $v_{i}<v_{i}^{\prime}$. Every element $g \in W(G)_{+}$is represented by a unique minimal word $\widetilde{g}$, which comes first in dictionary order among all words representing $g$.

A word $\widetilde{g}$ is minimal unless it contains a subword of the form $v_{i} \cdots v_{j} a$, such that $a<v_{i}$ and the letters $v_{i}, \ldots, v_{j}$ commute with $a$; cf. [GS, $\S 4$ ]. In this case, we could reorder the letters as $a v_{i} \cdots v_{j}$ to obtain a $\widetilde{g}^{\prime}<\widetilde{g}$ with $\left[\widetilde{g}^{\prime}\right]=[\widetilde{g}]$.

Here is an explicit finite-state automaton $\mathcal{A}$ which accepts exactly the minimal words. The states of $\mathcal{A}$ are given by

$$
\Sigma=\{S: V(G) \rightarrow\{0,1\}\} .
$$

In other words we have one bit for each generator $v$, and its value is recorded by $S(v)$. The initial and fail states are given by $S_{0}$ and $S_{1}$, where all bits are zero and all bits are one.

The automaton reads the letters of a word $\widetilde{g}=v_{1} \cdots v_{k}$, starting in state $S_{0}$, and makes transitions according to a function $\Sigma \times V(G) \rightarrow \Sigma$ which we will write as $(S, v) \mapsto S v$. The word is accepted unless $S_{0} v_{1} \cdots v_{k}=S_{1}$.

The transition function is defined as follows. First, we set $S v=S_{1}$ if $S(v)=1$. In other words, a word is rejected if the letter $v$ is read while its bit is set. Otherwise, we set $S v=S^{\prime}$ where

$$
S^{\prime}(a)= \begin{cases}1 & \text { if } v>a \text { and }[v, a]=1 \\ 0 & \text { if }[v, a] \neq 1, \text { and } \\ S(a) & \text { in all other cases }\end{cases}
$$


The bit $S^{\prime}(a)$ is set in the first case because we are in danger of forming a non-minimal subword $v \cdots a$; it is reset in the second case, because a letter not commuting with $a$ now prevents reordering. Thus one may easily verify:

The words accepted by $\mathcal{A}$ coincide with the minimal words for elements of $W(G)_{+}$.

Minimality is not local. We remark that minimality is not a local property the word, e.g. it is not just a condition on adjacent letters. For example, $G=A_{3}$ with consecutive vertices $(a, b, c)$, and we order the vertices so $a<b<c$, then $c a^{k}$ and $a^{k} b$ are minimal words but $c a^{k} b$ is not (it can be replaced by $\left.b c a^{k}\right)$.

The directed graph $\Delta$. We now define a directed graph $\Delta$ together with a map $\pi: E(\Delta) \rightarrow V(G)$. Let $V(\Delta)$ consist of the states $S \in \Sigma$ that can be reached from $S_{0}$, aside from the fail state $S_{1}$. Let

$$
E(\Delta)=\left\{\left(S, S^{\prime}, v\right): S, S^{\prime} \in V(\Delta) \text { and } S v=S^{\prime}\right\} .
$$

For $e=\left(S, S^{\prime}, v\right)$ we define $[e]=\left(S, S^{\prime}\right)$ and $\pi(e)=v$. In other words, the edge $e$ connects $S$ to $S^{\prime}$ and is labeled by $v$.

Theorem 4.2 The growth rate of $(G, w)$ is well-defined and satisfies

$$
\lambda(G, w)=\lambda(\Delta, w \circ \pi)
$$

for all positive weights $w$. The smallest positive zero of $Q(t)$ is given by $t=1 / \lambda(G, w)$.

Proof. By the properties of $\mathcal{A}$ just described, the map $\left(e_{1}, \ldots, e_{k}\right) \mapsto$ $\pi\left(e_{1}\right) \cdots \pi\left(e_{k}\right)$ yields a bijection:

$$
\begin{gathered}
\text { \{paths } \left.\left(e_{1}, \ldots, e_{k}\right) \text { in } \Delta \text { starting at } S_{0}\right\} \leftrightarrow \\
\left\{\text { minimal words } v_{1} \cdots v_{k} \text { in } W(G)_{+}\right\} .
\end{gathered}
$$

But the paths starting at $S_{0}$ grow at the same rate as all paths in $\Delta$, since every vertex can be reached from $S_{0}$. Thus $\lambda(G, w)$ is well-defined and coincides with $\lambda(\Delta, w \circ \pi)$. By the general results in $\S 2, \tau=1 / \lambda(G, w)$ gives the radius of convergence of the generating function $f(t)=1 / Q(t)$ defined by (4.2), and hence $\tau$ is also the smallest positive zero of $Q(t)$. 


\section{Basic properties and examples.}

1. Homogeneity. We have $\lambda(G, \alpha w)=\lambda(G, w)^{1 / \alpha}$.

2. Monotonicity. Let $H \subset G$ be an induced subgraph of $G$. Then $W(H)$ is a subgroup of $W(G)$, and hence

$$
\lambda(H, w \mid H) \leq \lambda(G, w)
$$

for all weights $w$. We also have

$$
\lambda(G, w) \leq \lambda\left(G, w^{\prime}\right)
$$

whenever $w(v) \geq w^{\prime}(v)$ for all $v \in V(G)$.

3. Joins. By the product formula (4.1), if $G=G_{1} * G_{2}$ then $Q=Q_{1} Q_{2}$, and hence

$$
\lambda(G, w)=\max \left(\lambda\left(G_{1}, w_{1}\right), \lambda\left(G_{2}, w_{2}\right)\right),
$$

where $w_{i}=w \mid V\left(G_{i}\right)$. The group $W(G)$ is simply the product $W\left(G_{1}\right) \times$ $W\left(G_{2}\right)$.

4. Complete graphs. Let $G=K_{n}$, with $V(G)=\left\{v_{1}, \ldots, v_{n}\right\}$ and $w_{i}=$ $w\left(v_{i}\right)$. Then $W(G) \cong \mathbb{Z}^{n}, \lambda(G, w)=1$, and $Q(t)=\prod_{1}^{n}\left(1-t^{w_{i}}\right)$.

5. Free groups. Let $G=n A_{1}$. Then $W(G) \cong F_{n}$ is the free group on $n$ generators, and $Q(t)=1-\sum_{1}^{n} t^{w_{i}}$. If all vertices are given weight one, then $Q(t)=1-n t$ and $\lambda(G, w)=n$ gives the usual growth rate of the free group on $n$ generators (the number of positive words of length $T$ grows like $n^{T}$ ).

6. The free group on two generators. We now specialize to the case $\left(G, w_{a}\right)$, where $G=2 A_{1}$ and $w_{a}$ gives the two vertices of $G$ weights 1 and $a>0$, and $W(G) \cong F_{2}$. As $a \rightarrow 0$ the growth rate of $W(G)_{+}$ tends to infinity, since the generator with weight $a$ can be repeated many times. In fact we have

$$
\lambda\left(G, w_{a}\right) \sim \frac{1}{|a \log a|} \quad \text { as } a \rightarrow 0 .
$$

This can be checked by setting $t=K a \log (1 / a)$ in the clique polynomial $Q(t)=1-t-t^{a}$, and then observing that the limit as $a \rightarrow 0$ changes sign at $K=1$. 
Connectivity and the reset code. Because of the behavior of joins (equation (4.6) above), for a general graph the function $h(w)=\log \lambda(G, w)$ need not be strictly convex, real-analytic or blow up at the boundary of the positive cone.

When $G^{\prime}$ is connected, however, $h(w)$ enjoys all three properties. To prove this we will construct a reset code $\widetilde{g}$ for the automaton underlying $\Delta$, i.e. a string of accepted letters that returns any state to the initial state $S_{0}$.

Theorem 4.3 If $G^{\prime}$ is connected, then there is an ordering for $V(G)$ such that $\Delta$ is strongly connected.

Proof. Let the ordered vertices of $G$ be $a_{1}<a_{2}<\cdots<a_{n}$. Since $G^{\prime}$ is connected, the ordering can be chosen so that the first $k$ vertices span a connected subgraph $G_{k}^{\prime}$ of $G^{\prime}$. Now construct a path in $G^{\prime}$ that tours all its vertices, starting at $a_{n}$, and stays in $G_{k}^{\prime}$ after its last visit to $a_{k}$. The vertices visited by $p$ define a word of the form

$$
\widetilde{g}=a_{n} w_{n-1} a_{n-1} w_{n-2} \cdots a_{2} w_{1} a_{1},
$$

whose adjacent letters do not commute, and such that the subword $w_{k}$ involves only the generators $a_{1}, \ldots, a_{k}$.

It is now easy to verify, using (4.3), that $S w=S_{0}$ for any state $S \in V(\Delta)$. First, observe that $S\left(a_{n}\right)=0$, since there is no way to set the last bit except by going to the fail state. Next, because adjacent letters of $w$ do not commute, we have $S w \neq S_{1}$, and hence $(S w)\left(a_{n}\right)=0$. Finally, for $k<n$, note that the last letter of $w_{k}$ does not commute with $a_{k}$, so it resets the $a_{k^{-}}$ bit to zero. All subsequent letters have indices $i \leq k$, so the $a_{k}$-bit remains at zero. Thus $(S w)\left(a_{k}\right)=0$ for all $k$, and hence $(S w)=S_{0}$.

Equivalently, $w$ defines a directed path from $S$ to $S_{0}$. Since we also have a directed path from $S_{0}$ to $S$ by the definition of $V(\Delta)$, the graph $\Delta$ is strongly connected.

Proof of Theorem 4.1. Since $G^{\prime}$ is connected, we may assume $\Delta$ is strongly connected. Then by Theorem $3.4, h(w)$ is a real-analytic, convex function of $w$.

Suppose $w_{n} \rightarrow w^{\prime}$ in the boundary of the cone of positive weights $P \subset$ $\mathbb{R}^{V(G)}$. Then $w^{\prime}\left(v_{1}\right)=0$ for some $v_{1} \in V(G)$. Since $G^{\prime}$ is connected and $|V(G)|>1$, there is a vertex $v_{2}$ such that $\left(v_{1}, v_{2}\right) \notin E(G)$. Let $H \cong 2 A_{1}$ be the induced subgraph of $G$ with $V(H)=\left\{v_{1}, v_{2}\right\}$, and note that $w_{n}\left(v_{1}\right) \rightarrow$ 0 while $\sup w_{n}\left(v_{2}\right)<\infty$. Thus $\lambda\left(H, w_{n} \mid H\right) \rightarrow \infty$ by homogeneity and equation (4.7), and hence $h\left(w_{n}\right)=\log \lambda\left(G, w_{n}\right) \rightarrow \infty$ by monotonicity. 
Now suppose there is a line $L$ such that $h$ is not strictly convex along the segment $L \cap P$. Since $h$ is convex and real-analytic, it must be linear on $L \cap P$. But this contradicts the fact that $h(w)$ tends to infinity at the boundary of $P$.

Proof of Theorem 1.3. Combine Theorems 3.3, 4.2 and 4.1.

\section{Cones and cohomology}

In this section we describe the relationship between the Perron and clique polynomials and the cohomology of $\Gamma$, and compare them to the Teichmüller polynomial of a fibered 3-manifold.

The positive cone. Let $\Gamma$ be a finite directed graph. Since the edges of $\Gamma$ are oriented, any closed path $p=\left(e_{1}, \ldots, e_{n}\right)$ determines a class $[p]=$ $\left[\sum e_{i}\right] \in H_{1}(\Gamma, \mathbb{Z})$. In particular, a simple curve $C$ determines a class $[C]$. A metric $m$ on $\Gamma$ determines a cohomology class $\phi_{m} \in H^{1}(\Gamma, \mathbb{R})$ by $\phi\left(\sum a_{i} e_{i}\right)=$ $\sum a_{i} m\left(e_{i}\right)$.

Theorem 5.1 Given $\phi \in H^{1}(\Gamma, \mathbb{R})$, the following conditions are equivalent:

1. $\phi$ can be represented by a metric.

2. $\phi([p])>0$ for any closed path $p$.

3. $\phi([C])>0$ for any simple curve $C$.

Proof. Clearly (1) $\Longrightarrow(2) \Longrightarrow(3)$. To see $(3) \Longrightarrow(2)$, follow a closed path $p$ until it first returns to one of the vertices it has previously visited. Then we can write $[p]=[C]+\left[p^{\prime}\right]$, where $[C]$ is a simple curve and $p^{\prime}$ is a shorter closed path. Thus $[p]=\sum\left[C_{i}\right]$ and $(3) \Longrightarrow(2)$. To see $(2) \Longrightarrow(1)$, consider the inclusion of cones

$$
K=C_{1}(\Gamma, \mathbb{R})_{+} \cap H_{1}(\Gamma, \mathbb{R}) \subset C_{1}(\Gamma, \mathbb{R})_{+},
$$

where $C_{1}(\Gamma, \mathbb{R})+=\mathbb{R}_{+}^{E(\Gamma)}$ is the positive orthant with respect to the basis coming from the edges. Then any class $\phi$ satisfying (2) gives a linear functional on $H_{1}(\Gamma, \mathbb{R})$ that is positive on $K$. By the geometric Hahn-Banach theorem it can be extended to a linear functional $m$ on $C_{1}(\Gamma, \mathbb{R})$ that is positive on $C_{1}(\Gamma, \mathbb{R})_{+}$, so it is represented by a metric. 
The classes $\phi$ satisfying these three conditions form the positive cone, which will be denoted by $H^{1}(\Gamma, \mathbb{R})_{+}$.

Expansion of cohomology classes. Since the Perron polynomial $P_{\Gamma}$ lies in $\mathbb{Z}\left[H_{1}(\Gamma)\right]$, the polynomial $P_{\Gamma}\left(t^{m}\right)$ and the expansion factor $\lambda(m)$ depend only on the cohomology class $\phi$ determined by $m$. In particular the expansion factor descends to a function on $H^{1}(\Gamma, \mathbb{R})_{+}$, which will be denoted by $\lambda(\Gamma, \phi)$.

Relation to the curve complex. Now let $G$ be the curve complex of $\Gamma$. Then each vertex $v$ of $G$ corresponds naturally to a simple curve $C$ on $\Gamma$. The map $v \mapsto[C]$ extends by linearity to a natural map

$$
\pi: C_{0}(G) \rightarrow H_{1}(\Gamma)
$$

and Theorem 1.4 can be restated as follows:

The natural map $\pi_{*}: \mathbb{Z}\left[C_{0}(G)\right] \rightarrow \mathbb{Z}\left[H_{1}(\Gamma)\right]$ sends the clique polynomial $Q_{G}$ to the Perron polynomial $P_{\Gamma}$.

Dualizing, we obtain a map

$$
\pi^{*}: H^{1}(\Gamma, \mathbb{R}) \rightarrow C^{0}(G, \mathbb{R}),
$$

and by condition (3) in Theorem 5.1 we can assert:

The positive cone $H^{1}(\Gamma, \mathbb{R})_{+}$is the preimage of the cone of positive weights $C^{0}(G, \mathbb{R})_{+}$under the natural map $\pi^{*}$.

Thus Theorem 4.1 implies:

Theorem 5.2 If $\Gamma$ is strongly connected, then $h(\phi)=\log \lambda(\Gamma, \phi)$ is a realanalytic, strictly convex function of $\phi$, and $h(\phi) \rightarrow \infty$ as $\phi$ approaches the boundary of $H^{1}(\Gamma, \mathbb{R})_{+}$.

It follows that $h(\phi)$ has a unique minimum under various convex constraints. For example, let us say $\phi$ is admissible if $0<\phi([C]) \leq 1$ for all multicurves $C$. Then we have:

Corollary 5.3 If $\Gamma$ is strongly connected, then there is a unique admissible cohomology class $\phi \in H^{1}(\Gamma, \mathbb{R})$ with minimum entropy.

This class can be realized by an admissible metric, but the latter is not unique in general.

Comparison with the Teichmüller polynomial. Infinite families of Perron-Frobenius matrices $A \in \mathrm{M}_{n}(\mathbb{Z})$ with $\rho(A)^{n}=O(1)$ (and $n \rightarrow \infty$ ) 
arise naturally by varying the metric $m$ (or the cohomology class $\phi$ ) on a fixed graph $\Gamma$. Their spectral radii are packaged by the polynomial

$$
P_{\Gamma} \in \mathbb{Z}\left[H_{1}(\Gamma)\right] .
$$

Similarly, infinite families of pseudo-Anosov maps $f \in \operatorname{Mod}\left(\Sigma_{g}\right)$ with $K(f)^{g}=O(1)$ (and $g \rightarrow \infty$ ) arise naturally from any fibered 3-manifold $M$ with $b_{1}(M)>1$. Their stretch factors $K(f)$ are packaged by the Teichmüller polynomial

$$
\Theta_{F} \in \mathbb{Z}\left[H_{1}(M) /(\text { torsion })\right]
$$

associated to a fibered face $F \subset H^{1}(M, \mathbb{R})$, introduced in $[\mathrm{Mc}]$. For each integral cohomology class $\phi$ in the convex cone

$$
\mathbb{R}_{+} \cdot F \subset H^{1}(M, \mathbb{R}),
$$

we obtain $f$ as the monodromy of a fibration $M \rightarrow S^{1}$ representing the map $\phi: \pi_{1}(M) \rightarrow \mathbb{Z}$, and $K(f)$ is the largest zero of the polynomial $\Theta_{F}\left(t^{\phi}\right)$. The function $h(\phi)=\log K(f)$ on the cone $\mathbb{R}_{+} \cdot F$ enjoys the same convexity and divergence properties as the function $h(\phi)$ on the positive cone $H^{1}(\Gamma, \mathbb{R})_{+}$.

Because of strict convexity, $h(\phi)$ achieves its minimum at a unique point $\phi \in F$. Examples where the minimizing $\phi$ has transcendental coordinates are given in [Sun]. In $\S 6$ we will see similar, but simpler, examples for graphs.

Further references. The Teichmüller polynomial can be defined in terms of the transition matrix for a train track associated to a fibration of $M$, which explains its connection to the Perron-Frobenius theory at hand; compare [Pen] and [HS]. For applications of $\Theta_{F}$ to the construction of mappingclasses with small entropy, see [Hir], [AD], [KT1], [FLM] and [KT2]. Recent generalizations of the Teichmüller polynomial to outer automorphisms of free groups are developed in $[\mathrm{AHR}]$ and $[\mathrm{DKL}]$.

\section{Graphs with small growth}

In this section we study the invariant $\lambda(G)$ and establish Theorems 1.5 and 1.6. We show $\lambda(G)$ is an algebraic number, and there are only finitely many graphs with $G^{\prime}$ connected and $1<\lambda(G)<M$; and for $M=8$, the list of such graphs is given by

$$
A_{2}^{*}, A_{2}^{* *}, A_{2}^{* * *}, A_{3}^{*}, Y^{*} \text { and } n A_{1},
$$

with $2 \leq n \leq 7$. For the proof we compute $\lambda(G)$ and the optimal weights on $V(G)$ in many concrete examples. 
Finiteness results. As in $\S 1$, we say a weight $w: G \rightarrow \mathbb{R}_{+}$is admissible if $w(K) \leq 1$ for all cliques of $G$, and we let

$$
\lambda(G)=\inf \{\lambda(G, w): w \text { is an admissible weight }\} .
$$

By equation (4.4) we have the useful monotonicity principle:

$$
\lambda(H) \leq \lambda(G) \text { if } H \text { is an induced subgraph of } G .
$$

We remark that the curve complex $G$ of $\Gamma$ generally has many subgraphs $H$ which do not come from subgraphs of $\Gamma$. This means we can obtain lower bounds of the form

$$
\lambda(H) \leq \lambda(G) \leq \lambda(\Gamma)
$$

may not be apparent from the structure of $\Gamma$ alone.

Theorem 6.1 Given $M>0$ there are only finitely many graphs $G$ with $G^{\prime}$ connected and $\lambda(G) \leq M$, up to isomorphism.

Proof. It suffices to show that $\lambda(G)$ is large whenever $|V(G)|$ is large. By Ramsey theory (see e.g. [Bol, $\S \mathrm{VI}]$ ), if $|V(G)| \geq\left(\begin{array}{c}2 n-2 \\ n-1\end{array}\right)$, either $G$ or $G^{\prime}$ contains a clique with $|K|=n$. If $K$ is a clique of $G^{\prime}$ then $G$ contains $n A_{1}$ as an induced subgraph, so $\lambda(G) \geq \lambda\left(n A_{1}\right)=n$.

Now suppose $K$ is a clique of $G$. The optimal weight $w$ for $G$ satisfies $w(K) \leq 1$, so $w(a) \leq 1 / n$ for some $a \in K$. Since $G^{\prime}$ is connected, there is a vertex $b \in V(G)$ that is not connected to $a$, and $w(b) \leq 1$. Let $H$ be the induced subgraph with $V(H)=\{a, b\}$. Then

$$
\lambda(G)=\lambda(G, w) \geq \lambda(H, w \mid H) \geq \lambda_{n},
$$

where $t=1 / \lambda_{n}$ is the smallest root of the clique polynomial $Q(t)=1-$ $t^{1 / n}-t$. We have already seen that $\lambda_{n} \rightarrow \infty$ like $n / \log n$ (equation (4.7)), so the proof is complete.

Since $\lambda(\Gamma) \geq \lambda(G)$, the same type of result holds for directed graphs.

Proposition 6.2 Let $\Gamma$ be a strongly connected directed graph with curve complex $G$. Then $|V(G)| \geq 1+|\chi(\Gamma)|$.

Proof. Since $\Gamma$ is strongly connected, we can write $\Gamma=\Gamma_{0} \cup \Gamma_{1} \cup \cdots \cup \Gamma_{n}$ where $\Gamma_{0}$ is a loop, each $\Gamma_{i}$ is strongly connected, and $\Gamma_{i}$ is obtained from $\Gamma_{i-1}$ by adding a directed arc $A_{i}$ between two vertices (or a loop based at one). Then $n=|\chi(\Gamma)|$, and there is at least one simple curve in $\Gamma_{i}$ through $A_{i}$, so $|V(G)| \geq n+1$. 
Corollary 6.3 There are only finitely many strongly connected graphs $\Gamma$ with no vertices of degree two and a given curve complex $G$.

Corollary 6.4 Given $M>1$, there are only finitely many strongly connected graphs with no vertices of degree two and $\lambda(\Gamma) \leq M$.

Example. Start with a directed loop with cyclically ordered vertices $\left(v_{1}, \ldots, v_{2 n}\right)$, and add a directed edge from $v_{i}$ to $v_{2 n-i}$ for $i=1,2, \ldots, n$. The result is a directed graph with exactly $n+1$ simple curves such that $|\chi(\Gamma)|=n$. Thus the bound in Proposition 6.2 is sharp.

Optimal weights. An admissible weight $w$ is optimal if $\lambda(G)=\lambda(G, w)$. It is symmetric if it is invariant under $\operatorname{Aut}(G)$, and maximal if there is no other admissible weight with $w^{\prime}(v) \geq w(v)$ for all $v$.

We say $G$ is a cone if it is isomorphic to a graph of the form $A_{1} * H$.

Theorem 6.5 If $G$ is not a cone, then $G$ carries a unique optimal weight. This weight is maximal and symmetric.

Proof. First suppose $G^{\prime}$ is connected and $|V(G)|>1$. Then $h(w)=$ $\log \lambda(G, w)$ is strictly convex and tends to infinity at the boundary of the positive cone, by Theorem 4.1. The admissibility conditions are convex and bound $h(w)$ from below, so there is a unique optimal weight.

In general, $G$ factors canonically as a join $G_{1} * \cdots * G_{n}$ with $G_{i}^{\prime}$ connected for all $i$, and we have

$$
\lambda(G, w)=\max \lambda\left(G_{i}, w \mid G_{i}\right) .
$$

Since $G$ is not a cone, $\left|V\left(G_{i}\right)\right|>1$ for all $i$, so each $G_{i}$ has a unique optimal weight $w_{i}$. Then the optimal weight for $G$ is the simply the unique convex combination $w=\sum \alpha_{i} w_{i}$ such that

$$
\lambda\left(G_{i}, w \mid G_{i}\right)=\lambda\left(G_{i}\right)^{1 / \alpha_{i}}=\lambda\left(G_{j}\right)^{1 / \alpha_{j}}
$$

for all $i, j$.

By convexity and uniqueness, the optimal weight $w$ must be maximal and symmetric. 
The case of a cone. The proof shows any join satisfies

$$
\lambda\left(G_{1} * G_{2}\right)=\lambda\left(G_{1}\right) \lambda\left(G_{2}\right) .
$$

Moreover, if $G=A_{1} * H$ and $\lambda(H)>1$, then $G$ has no (positive) optimal weight; the minimum of $\lambda(G, w)$ is 'attained' at the boundary of the positive cone, by a weight with $w(v)=0$ on the $A_{1}$ factor. (If we allow vanishing mass, then any graph has an optimal weight, and it is unique unless $G \cong$ $K_{n}$.)

Values of $\lambda(G)$. Theorem 6.5 allows one to compute $\lambda(G)$ in many cases. Here is a list of useful examples, including all the graphs shown in Figure 1.

1. We have $\lambda\left(n A_{1}\right)=n$. By symmetry the optimal weight $w$ must be constant, and by maximality its constant value must be 1 . The clique polynomial for this weight is $Q(t)=1-n t$, with $t=1 / n$ as its smallest positive root.

2. We have $\lambda\left(A_{3}\right)=\lambda\left(A_{1} * A_{2}\right)=2$ by equation (6.2). The optimal 'weight' vanishes on the $A_{1}$ factor.

3. We have $\lambda(G) \geq 2$ whenever $G^{\prime}$ is connected and $|V(G)|>1$. Indeed, in this $G$ contains an induced subgraph of $H \cong 2 A_{1}$; apply equation (6.1).

4. We have $\lambda\left(K_{n}\right)=1$ (for $n>0$ ). The group $W\left(K_{n}\right) \cong \mathbb{Z}^{n}$ has polynomial growth, so $\lambda\left(K_{n}, w\right)=1$ for all $w$.

5. We have $\lambda\left(K_{n}^{*}\right)=2^{n}$. The optimal weights are $1 / n$ on the vertices of $K_{n}$ and 1 on the remaining vertex; thus $Q(t)=\left(1-t^{1 / n}\right)^{n}-t$.

6. We have $\lambda\left(A_{2}+n A_{1}\right)=(1+\sqrt{n})^{2}$. Here the optimal weight is given by $w(v)=1 / 2$ for $v \in V\left(A_{2}\right)$ and $w(v)=1$ for $v \in V\left(n A_{1}\right)$; thus $Q(t)=1-2 t^{1 / 2}-(n-1) t$.

7. We have $\lambda\left(2 A_{2}\right)=6+4 \sqrt{2}$. By symmetry the optimal weights are all $1 / 2$; thus $Q(t)=1-4 t^{1 / 2}+2 t$.

8. $G=L_{n}$. For a loop with $n \geq 4$ vertices, the optimal weights are all $1 / 2$ and $Q(t)=1-n t^{1 / 2}+n t$. This gives $\lambda\left(L_{4}\right)=4, \lambda\left(L_{5}\right)=10 /(3-\sqrt{5})$, etc.

9. We have $\lambda\left(L_{n}^{*}\right)=(n-1)^{2}$ for $n \geq 4$. The optimal weights are given by $w(v)=1 / 2$ along the loop and by $w(v)=1$ at the remaining vertex; thus $Q(t)=1-n t^{1 / 2}+(n-1) t$. 
10. We have $\lambda\left(L_{3}^{*}\right)=8$. The case $n=3$ is exceptional, since we have a clique of size 3 ; thus $w(v)=1 / 3$ along $L_{3}$, and $Q(t)=1-3 t^{1 / 3}+$ $3 t^{2 / 3}-2 t$.

11. We have $\lambda\left(A_{4}\right)=8$. In this case by symmetry and maximality there is an $a \in(0,1)$ such that the optimal weights on the consecutive vertices of $A_{4}$ are $(a, 1-a, 1-a, a)$. Hence

$$
Q(t)=1-2 t^{a}-2 t^{1-a}+t^{2-2 a}+2 t .
$$

To determine the value of $a$, we observe that both $Q$ and $d Q / d a$ must vanish at $(a, \tau)$; hence $a=2 / 3$ and $\tau=1 / 8$.

12. Transcendental weights. For $G=A_{3}^{*}$ we have $\lambda=\lambda(G)=3+2 \sqrt{2}$. This is the simplest example of a graph whose optimal weight $w$ is not rational. Its values can be determined as follows. By symmetry and maximality, there is an $a \in(0,1)$ such that $w$ takes the values $(a, 1-a, a)$ along the vertices of $A_{3}$, and $w(v)=1$ at the remaining vertex of $G$. Thus the clique polynomial has the form

$$
Q(t)=1-2 t^{a}-t^{1-a}+t .
$$

Now by optimality we must have $d Q / d a=0$ at $t=\tau=1 / \lambda$. This gives a second relation $2 \tau^{a}=\tau^{1-a}$; when combined with $Q(\tau)=0$, it implies $\lambda=3+2 \sqrt{2}$ and $a=\log (2 \lambda) / \log \left(\lambda^{2}\right)=0.69661 \ldots$ In particular, $a$ is transcendental (see e.g. [Gel, Thm II, p.106]). The graphs $G=A_{3}+n A_{1}$ can be analyzed in the same way; the value of $a$, as a function of $\lambda$, is independent of $n$.

13. More generally, we have $\lambda\left(K_{p, q}+n A_{1}\right)=(\sqrt{n}+\sqrt{p q})^{2}$. Since $K_{1,2} \cong$ $A_{3}, K_{1,3} \cong Y$ and $K_{1,4} \cong X$, this formula covers the remaining graphs in Figure 1.

As in the previous case, here the optimal weight $w$ depends on a value $a$ which can be computed using the relation $d Q / d a=0$. Writing $K_{p, q}=\left(p A_{1}\right) *\left(q A_{1}\right)+n A_{1}$, by symmetry and maximality there is an $a \in(0,1)$ such that $w(v)=a, 1-a$ and 1 on the factors $p A_{1}, q A_{1}$ and $n A_{1}$ respectively. Thus by (4.1) we have

$$
Q(t)=\left(1-p t^{a}\right)\left(1-q t^{1-a}\right)-n t .
$$

The relation $d Q / d a=0$ gives $p t^{a}=q t^{1-a}=\sqrt{p q t}$ at $t=\tau$, and hence $\tau=\lambda^{-1}$ is the smallest positive zero of the equation $Q(\tau)=$ $(1-\sqrt{p q \tau})^{2}-n \tau=0$. 
Again, the optimal weights are transcendental for typical values of $p$ and $q$. For example, $a=\log (p) / \log (q p)$ for the complete bipartite graph $K_{p, q}$.

Algebraic properties. In contrast to the behavior of the optimal weights, we have:

Theorem 6.6 For any graph $G$, the growth rate $\lambda(G)$ is algebraic over $\mathbb{Q}$.

Sketch of the proof. Introduce new variables $u_{i}=t^{w\left(v_{i}\right)}$, where $V(G)=$ $\left\{v_{1}, \ldots, v_{n}\right\}$. Then $Q\left(t^{w}\right)=Q\left(u_{1}, \ldots, u_{n}\right)$ gives a polynomial function on $\mathbb{R}^{n}$ with integer coefficients. The optimal weight $w$ lies in the interior of a facet of the convex set of admissible weights, defined by linear equations of the form $\sum_{i \in C} w\left(v_{i}\right)=1$. In $u$-coordinates, these linear equations give an algebraic subvariety of $\mathbb{R}^{n} \times \mathbb{R}$ defined by equations of the form $\prod_{i \in C} u_{i}=t$. Furthermore $Q\left(u_{1}, \ldots, u_{n}\right)=0$. Since $t$ is locally maximized, subject to the condition that it is a zero of $Q\left(t^{w}\right)$, we have additional equations of the form $X Q=0$, with $X$ a rational linear combination of $d / d u_{i}$. Altogether these equations cut out a real algebraic subvariety $V \subset \mathbb{R}^{n} \times \mathbb{R}$, defined over $\mathbb{Q}$, such that $1 / \lambda(G)$ is the projection of one of its components to the $t$-coordinate. Thus $\lambda(G)$ is algebraic over $\mathbb{Q}$.

In fact the same argument shows the optimal weights satisfy $\lambda(G)^{w\left(v_{i}\right)} \in \overline{\mathbb{Q}}$ for all $i$.

Classification. We now proceed to the enumeration of graphs with $G^{\prime}$ connected and $1<\lambda(G)<8$.

The distance $d(x, y)$ between two vertices $x, y \in V(G)$ is the minimal number of edges in a path connecting them (or $\infty$ if none exists). The diameter of $G$, denoted $\operatorname{diam}(G)$, is the maximum distance between a pair of its vertices.

Lemma 6.7 Let $G$ be a graph such that both $G$ and $G^{\prime}$ are connected. Then either $G \cong A_{1}$, or $G$ contains an induced subgraph isomorphic to $A_{4}, 2 A_{2}$ or $L_{3}^{*}$.

Proof. First suppose $G$ is bipartite; that is, suppose we have a partition of $V(G)$ into disjoint sets $A$ and $B$ such that all edges run between $A$ to $B$. Let $B(a)$ denote the subset of $B$ at distance 1 from $a \in A$. Since $G$ is connected, $B(a)$ is nonempty and $B=\bigcup B(a)$ (unless $G \cong A_{1}$ ). If $B(a)=B$ for all $a$, then $G$ is a complete bipartite graph, contrary to our assumption that $G^{\prime}$ 
is connected. Thus $B(a) \neq B\left(a^{\prime}\right)$ for some $a, a^{\prime} \in A$. If both $B(a)-B\left(a^{\prime}\right)$ and $B\left(a^{\prime}\right)-B(a)$ are nonempty, then we obtain an induced copy of $2 A_{2}$. Otherwise, we may assume $B(a) \subset B\left(a^{\prime}\right)$. Then we can find $b, b^{\prime} \in B$ such that $\left(a, b, a^{\prime}, b^{\prime}\right)$ is a path in $G$ but there is no edge from $a$ to $b^{\prime}$. Then these vertices span a copy of $A_{4}$.

Now suppose $G$ is not bipartite. Then $G$ contains a closed path of odd length. The shortest path of this type gives an induced copy of $L_{n}$ in $G$. If $n \geq 5$ then $L_{n}$ contains an induced copy of $A_{4}$. So we may assume $n=3$; in other words, we may assume $G$ contains a triangle, say with vertices $\{a, b, c\}$.

If $\operatorname{diam}\left(G^{\prime}\right) \geq 3$ then a minimal path joining 2 vertices with $d(x, y)=3$ gives a copy of $A_{4}$ in $G^{\prime}$. But $A_{4}^{\prime} \cong A_{4}$, so we obtain a copy of $A_{4}$ in $G$.

Thus we may assume $\operatorname{diam}\left(G^{\prime}\right)=2$. In particular, $d(b, c)=2$, so we have a path of the form $\left(b, a^{\prime}, c\right)$ in $G^{\prime}$. If $\left(a, a^{\prime}\right) \notin E(G)$, then the vertices $\left\{a, b, c, a^{\prime}\right\}$ span a copy of $L_{3}^{*}$ in $G$ and we are done. So we may assume $\left(a, a^{\prime}\right) \in E(G)$. Similarly, we may assume we have vertices $b^{\prime}$ and $c^{\prime}$ such that $\left(a, a^{\prime}\right),\left(b, b^{\prime}\right)$ and $\left(c, c^{\prime}\right)$ are edges of $G$, and no other edges join $\left\{a^{\prime}, b^{\prime}, c^{\prime}\right\}$ to $\{a, b, c\}$. See Figure 4 .

To complete the proof, observe that if $\left(a^{\prime}, b^{\prime}\right) \in E(G)$, then the path $\left(a^{\prime}, b^{\prime}, b, c\right)$ gives a copy of $A_{4}$ in $G$; while if $\left(a^{\prime}, b^{\prime}\right) \notin E(G)$, then the path $\left(a^{\prime}, a, b, b^{\prime}\right)$ does the same.

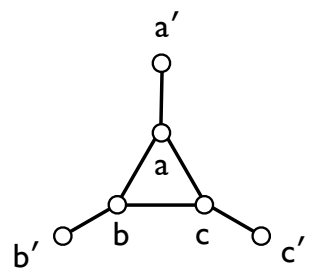

Figure 4. A triangle with legs.

Remark. There are many interesting graphs with $\operatorname{diam}(G)=\operatorname{diam}\left(G^{\prime}\right)=$ 2. For example, suppose $A \subset \mathbb{Z} / n$ satisfies $A=-A, A+A=\mathbb{Z} / n$, and $|A|+|A|<n$. (Such $A$ are easily found when $n$ is large.) Let $G$ be the graph with $V(G)=\mathbb{Z} / n$ whose edges connect $x$ to $y$ iff $x \in y+A$. Since $x+A+A=V(G)$, we have $\operatorname{diam}(G)=2$, and since $|A|+|A|<n$, we have $\operatorname{diam}\left(G^{\prime}\right)=2$ as well.

Lemma 6.8 Let $G_{0}$ be a connected graph. Then either $G_{0} \cong A_{1}, A_{2}, A_{3}$ or $Y$, or $G_{0}$ contains $A_{4}, X, L_{3}$ or $L_{4}$ as an induced subgraph. 
Proof. Suppose $G_{0}$ does not contain $L_{3}, L_{4}$ or $A_{4}$ as induced subgraph. Then $G_{0}$ cannot contain $L_{n}$ for $n \geq 5$, so it is a tree. We have $\operatorname{diam}\left(G_{0}\right) \leq 2$, since otherwise $G_{0}$ contains $A_{4}$. It follows that $G_{0}$ is a cone of the form $A_{1} *\left(k A_{1}\right)$ for some $k$. The cases $k=0,1,2,3$ are the graphs given, and any larger cone contains a copy of the graph $X$.

Proof of Theorem 1.6. Suppose $1<\lambda(G)<8$ and $G^{\prime}$ is connected. Then $G \neq A_{1}$. We have seen that $\lambda(H) \geq 8$ for $H=A_{4}, L_{3}^{*}$ and $2 A_{2}$, so these $H$ cannot be induced subgraphs of $G$. Thus $G$ is disconnected, by Lemma 6.7. If $G$ has more than two nontrivial components, then it contains a copy of $2 A_{2}$; but $\lambda\left(2 A_{2}\right)=8$, so this is impossible. Thus $G=G_{0}+k A_{1}$ for some connected graph $G_{0}$, and $k \geq 1$. Since $\lambda\left(H^{*}\right) \geq 8$ for $H=A_{4}, X, L_{3}$ and $L_{4}$, the Lemma above implies $G_{0}=A_{1}, A_{2}, A_{3}$ or $Y$. For each of these we can bound the value of $k$ using monotonicity and the explicit values of $\lambda(G)$ summarized in Figure 1. The graphs which remain are those listed in the statement of the Theorem.

Questions. Is $\lambda(G) \geq|V(G)|$, provided $G^{\prime}$ is connected? If $G$ contains a clique of order $n$ and $G^{\prime}$ is connected, is $\lambda(G) \geq 2^{n}$ ?

\section{Reciprocal matrices}

In this section we prove Theorem 1.1 on Perron-Frobenius matrices, along with the following complementary result.

Theorem 7.1 For any non-negative, irreducible, reciprocal matrix $A \in$ $\mathrm{M}_{n}(\mathbb{Z}), n \geq 2$, we have

$$
\rho(A)^{n} \geq \gamma^{4}
$$

Here $\gamma=(1+\sqrt{5}) / 2$ denotes the golden mean.

Matrices. Let $A \geq 0$ be the adjacency matrix of a directed graph $\Gamma$ with curve complex $G$, and let $n=|V(\Gamma)|$ be the rank of $A$. We say $A$ and $\Gamma$ are reciprocal if the characteristic polynomial $\operatorname{det}(t I-A)$ is reciprocal. Recall from equation (1.3) we have

$$
\rho(A)^{n}=\lambda(\Gamma, 1)^{n} \geq \lambda(\Gamma) \geq \lambda(G) .
$$

To illustrate the approach, we first prove the following result (which does not assume that $A$ is reciprocal). 
Theorem 7.2 The minimum of $\rho(A)$ over all irreducible $A \in \mathrm{M}_{n}(\mathbb{Z}), n \geq$ 2 , is given by $2^{1 / n}$. If we require $A$ is Perron-Frobenius, then the minimum of $\rho(A)^{n}$ is given by the largest root of the polynomial

$$
t^{n}-t-1 \text {. }
$$

The first statement appears in [Pen, p. 445]. For a conjectural lower bound on $\rho(A)$ in terms of $\operatorname{deg}(\rho(A) / \mathbb{Q})$, see [B, Conj. D].

Proof. First assume $A$ is irreducible, with associated directed graph $\Gamma$ and curve complex $G$. Then $\Gamma$ is strongly connected, $G^{\prime}$ is connected, and $\lambda(G)>1$; hence $\lambda(G) \geq 2$, which gives $\rho(A)^{n} \geq 2$. To see equality can hold, let $\Gamma$ be the graph obtained by replacing one edge of $L_{n}$ with two parallel edges. Then $\Gamma$ has two simple curves, each of length $n$, so $G=2 A_{1}$ and $P(t)=1-2 t^{n}$, which gives $\rho(A)^{n}=2$.

Now suppose $A$ is a Perron-Frobenius matrix. Since $P(t)=\operatorname{det}(t I-A)$ has a unique largest root, when $G=2 A_{1}$ we cannot have $P(t)=t^{n}-2$; the best approximation that can arise is $t^{n}-t-1$, whose largest root $\nu_{n}$ is less than 3 and thus provides a lower bound for $\rho(A)$ (since $\lambda(G) \geq 3$ for the other graphs appearing in Theorem 1.6). This lower bound is achieved by the graph $\Gamma$ obtained from $L_{n}$ by adding an edge between its first and third vertices, to yield a second simple curve of length $n-1$ (see Figure 5).

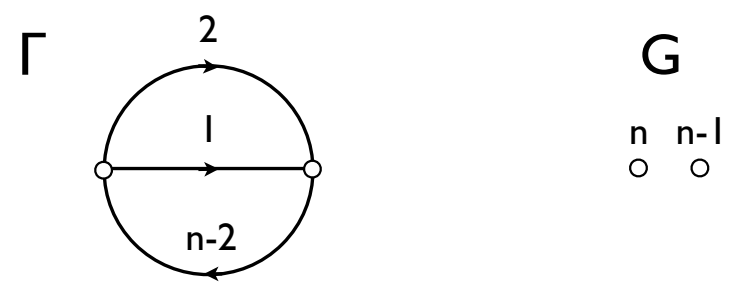

Figure 5. Realizing $\operatorname{det}(t I-A)=t^{n}-t-1$.

Algebraic integers. We now turn to the reciprocal case. Let $L_{2 g}(t)$ be the polynomial given in equation (1.1), and let $\mu_{2 g}$ denote the largest positive root of the polynomial (with rational exponents)

$$
L_{2 g}\left(t^{1 / 2 g}\right)=1-t^{\frac{1}{2}-\frac{1}{2 g}}-t^{\frac{1}{2}}-t^{\frac{1}{2}+\frac{1}{2 g}}+t .
$$

Then $\mu_{4} \geq \mu_{6} \geq \mu_{8} \cdots$ and $\lim _{g \rightarrow \infty} \mu_{2 g}=\gamma^{4}$, the largest positive root of the polynomial $1-3 t^{1 / 2}+t$. We note that $\mu_{12}^{1 / 12}$ is Lehmer's number, the smallest known Salem number. 
Reciprocal weights. Let $P(t)=\sum_{1}^{n} a_{i} t^{\alpha_{i}}$ be a polynomial in $\mathbb{Z}\left[t^{\alpha}: \alpha>\right.$ $0]$. Its degree and its reciprocal polynomial are defined by

$$
\operatorname{deg}(P)=\max \alpha_{i} \quad \text { and } \quad P^{*}(t)=t^{\operatorname{deg}(P)} P\left(t^{-1}\right) .
$$

We say $P(t)$ itself is reciprocal if $P(t)= \pm P^{*}(t)$; it is symmetric if the sign is positive, and otherwise antisymmetric. The zeros of a reciprocal polynomial are invariant under $t \mapsto 1 / t$.

We say a weight $w$ for $G$ is reciprocal if $Q_{G}\left(t^{w}\right)$ is a reciprocal polynomial, and we let

$$
\lambda_{\text {rec }}(G)=\inf \{\lambda(G, w): w \text { is admissible and reciprocal }\} .
$$

When $A$ is reciprocal, with directed graph $\Gamma$ and curve complex $G$, equation (1.3) can be replaced by

$$
\rho(A)^{n} \geq \lambda_{\text {rec }}(G) .
$$

Optimal reciprocal weights. The value of $\lambda_{\text {rec }}(G)$ can be computed by the same methods used to compute $\lambda(G)$ in $\S 6$. The main difference is that the reciprocal admissible weights are a finite union of convex sets, $W_{\text {rec }}=$ $\bigcup_{1}^{n} W_{i}$. The different components come from different ways of pairing the monomials in $Q_{G}\left(t^{w}\right)$ to make it symmetric. Minimization of $\lambda(G, w)$ for $w \in W_{i}$ amounts to maximizing the smallest root of a polynomial $P_{i}(t)$ with variable exponents, and again the principles of symmetry and maximality from $\S 6$ apply.

Theorem 7.3 If $G^{\prime}$ is connected and $|V(G)|>1$, then $\lambda_{\text {rec }}(G) \geq \gamma^{4}$.

For the proof, we first compute $\lambda_{\text {rec }}(G)$ for the graphs $n A_{1}, A_{2}^{*}, A_{2}^{* *}$, $A_{2}^{* * *}, A_{3}^{*}$ and $Y^{*}$ with $\lambda(G)<8$.

1. If $Q_{G}\left(t^{w}\right)$ is antisymmetric, then $Q_{G}(1)=\sum_{K}(-1)^{|K|}=0$. This means the cell complex $X_{G}$ obtained from $G$ by adding a $k$-cell for each clique with $|K|>2$ has Euler characteristic 1.

2. If $1<\lambda(G)<8$ and $G^{\prime}$ is connected, then any reciprocal weight gives a symmetric polynomial, since the graphs listed in Theorem 1.6 all have $\chi\left(X_{G}\right) \geq 2$.

3. We have $\lambda_{\text {rec }}\left(n A_{1}\right)=\infty$ for $n \geq 2$, since there are no reciprocal weights. 
4. We have $\lambda_{\text {rec }}\left(A_{2}^{*}\right)=\gamma^{4} \approx 6.85410$. To see this, let $w$ be a weight on $G=A_{2}^{*}$ with values $a, b$ on the vertices of $A_{2}$ and $c$ on the remaining vertex; then

$$
Q_{G}\left(t^{w}\right)=1-t^{a}-t^{b}-t^{c}+t^{a+b} .
$$

To make this polynomial symmetric and degree one, we must impose the conditions $a+b=1$ and $c=1 / 2$, which gives a polynomial of the form

$$
P_{1}(t)=1-t^{a}-t^{1 / 2}-t^{1-a}+t
$$

with $0<a<1$. The smallest positive root of $P_{1}(t)$ is maximized when $a=1 / 2$, which gives $P_{1}(t)=1-3 t^{1 / 2}+t$ and $\inf _{W_{1}} \lambda(G, w)=$ $\lambda_{\text {rec }}(G)=\gamma^{4}$.

5. We have $\lambda_{\text {rec }}\left(A_{3}^{*}\right)=\gamma^{4}$ as well. In this case two families of symmetric polynomials arise: the family given in equation (7.1), and the family

$$
P_{2}(t)=1-t^{a}-t^{1 / 2-a}+t^{1 / 2}-t^{1 / 2+a}-t^{1-a}+t
$$

with $a \in(0,1 / 2)$. The smallest positive root of the polynomial above is maximized when $a=1 / 4$, giving $P_{2}(t)=1-2 t^{1 / 4}+t^{1 / 2}-2 t^{3 / 4}+t$ and $\inf _{W_{2}} \lambda(G, w) \approx 12.577>\gamma^{4}$, so the second polynomial does not change the value of $\lambda_{\text {rec }}(G)$.

6. We have $\lambda_{\text {rec }}\left(A_{2}^{* *}\right)=(2+\sqrt{3})^{2} \approx 13.92820$. The minimum value of $\lambda(G, w)$ is attained by the constant weight $w(v)=1 / 2$, which gives $Q_{G}\left(t^{w}\right)=1-4 t^{1 / 2}+t$

7. Similarly $\lambda_{\text {rec }}\left(A_{2}^{* * *}\right)=(5+\sqrt{21})^{2} / 4 \approx 22.9564$, coming from $Q_{G}\left(t^{w}\right)=$ $1-5 t^{1 / 2}+t$

8. We have $\lambda_{\text {rec }}\left(Y^{*}\right)=\mu_{4} \approx 8.79462$. To see this, let $(a, b, c, d, e)$ denote the values of $w(v)$, suitably ordered; then

$$
Q_{G}\left(t^{w}\right)=1-t^{a}-t^{b}-t^{c}-t^{d}-t^{e}+t^{a+b}+t^{a+c}+t^{a+d} .
$$

By imposing relations to make $Q_{G}\left(t^{w}\right)$ symmetric and degree 1 , we obtain the following 4 families of polynomials:

$$
\begin{aligned}
& P_{1}(t)=1-t^{a}-t^{1 / 2}-t^{1-a}-t \\
& P_{2}(t)=1-t^{a}-t^{1 / 2-a}+t^{1 / 2}-t^{1 / 2+a}-t^{1-a}+t \\
& P_{3}(t)=1-t^{a}-t^{1 / 2-2 a}+t^{1 / 2}-t^{1 / 2+2 a}-t^{1-a}+t \\
& P_{4}(t)=1-t^{a}-t^{1 / 2-2 a}+t^{1 / 2-a}-t^{1 / 2}+t^{1 / 2+a}-t^{1 / 2+2 a}-t^{1-a}+t .
\end{aligned}
$$


Here $0<a \leq 1 / 4$ for $P_{1}$ and $P_{2}$ and $0<a<1 / 4$ for $P_{3}$ and $P_{4}$. (The first family, for example, arises by imposing the conditions $a+d=1$, $b=1 / 2, a+b=c$ and $a+c=e$, which makes $e=2 a+1 / 2$. For this weight to be admissible we need $e \leq 1$ and hence $a \leq 1 / 4$.)

One can now readily check that the minimum of $\lambda(G, w)$ arises from $P_{1}(t)$ with $a=1 / 4$, which gives

$$
P_{1}(t)=1-t^{1 / 4}-t^{1 / 2}-t^{3 / 4}+t
$$

and hence $\lambda(G, w)=\mu_{4}$.

Proof of Theorem 7.3. We have $\lambda_{\text {rec }}(G) \geq \lambda(G) \geq 8$ except for the graphs listed in Theorem 1.6, and we have just seen that those satisfy $\lambda_{\text {rec }}(G) \geq \gamma^{4}$.

Proof of Theorem 7.1. Let $A \in \mathrm{M}_{n}(\mathbb{Z})$ be an irreducible reciprocal matrix with $\rho(A)>1$. Let $G$ be the curve complex of the associated directed graph $\Gamma$; then $G^{\prime}$ is connected, and $|V(G)|>1$, so Theorem 7.3 implies

$$
\rho(A)^{n}=\lambda(\Gamma, 1)^{n} \geq \lambda_{\text {rec }}(G) \geq \gamma^{4} .
$$

Sharpness and parity. The metrized directed graph $\Gamma$ at the left in Figure 6 gives an adjacency matrix $A \in \mathrm{M}_{2 g}(\mathbb{Z})$ with $\operatorname{det}(t I-A)=t^{2 g}-3 t^{g}+1$ for every $g \geq 2$. (When $g=2$, two vertices in the graph are identified.) For $g=1$ we may take $A=\left(\begin{array}{ll}2 & 1 \\ 1 & 1\end{array}\right)$. Thus Theorem 7.1 is sharp when $n=2 g$ is even.

When $n$ is odd, Theorem 7.1 can be improved at least to $\rho(A)^{n} \geq 8$. In this case the graphs $A_{2}^{*}$ and $A_{3}^{*}$ cannot occur, because of the $t^{1 / 2}$ terms in equations (7.1) and (7.2); and the remaining graphs satisfy $\lambda_{\text {rec }}(G) \geq 8$.

Proof of Theorem 1.1. Let $A \in \mathrm{M}_{2 g}(\mathbb{Z}), g>1$, be a reciprocal PerronFrobenius matrix, and let $R(t)=1-3 t^{g}+t^{2 g}$. Then $P(t)=\operatorname{det}(t I-A)$ has a unique root of maximum modulus, so we cannot have $P(t)=R(t)$. If we repeat the analysis of Theorem 7.1 with $R(t)$ removed the competition, we find that the best approximation to $R(t)$ which arises is given by $L_{2 g}(t)$. This yields the lower bound $\rho(A)^{2 g} \geq \mu_{2 g}$. (We have $\mu_{2 g}<8$ for $g>2$; the case $g=2$ with $\mu_{2 g}=8.794 \ldots$ can be checked directly.) This bound is achieved (for each $g \geq 2$ ) by the family of graphs shown at the right in Figure 6. These corresponding adjacency matrices are Perron-Frobenius because $\operatorname{gcd}(g, g+1)=1$. 
$\Gamma$
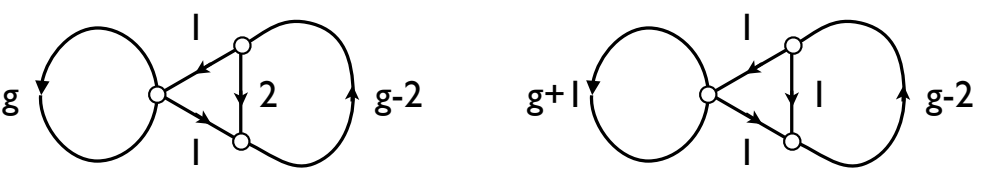

G

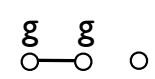

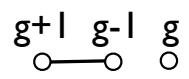

Figure 6. Realizing the polynomials $t^{2 g}-3 t^{g}+1$ and $t^{2 g}-t^{g}\left(1+t+t^{-1}\right)+1$ by directed graphs.

\section{A Appendix: Optimal metrics and outer space}

In this Appendix we connect the results of $\S 5$ on directed graphs with the results of Lim and Kapovich-Nagnibeda on optimal metrics for undirected graphs $[\mathrm{Lim}],[\mathrm{KN}]$. By including strict convexity in the picture, we obtain a simple description of entropy as a piecewise-smooth Morse function on Culler-Vogtmann's outer space [CV]. For related results, see [PS].

Optimal metrics. Let $G$ be a finite, connected, undirected graph, possibly with loops and parallel edges. Assume all vertices $p \in V(G)$ have degree $d(p) \geq 3$. We define the entropy and volume of a metric $m: E(G) \rightarrow \mathbb{R}_{+}$by

$$
\operatorname{vol}(G, m)=\sum_{e \in E(G)} m(e)
$$

and

$$
h(G, m)=\lim _{T \rightarrow \infty} \log N_{0}(T)^{1 / T},
$$

where $N_{0}(T)$ is the number of closed geodesics in $(G, m)$ of length $\leq T$. Let

$$
H(G)=\inf _{m} \operatorname{vol}(G, m) h(G, m) .
$$

We say $m$ is optimal if it achieves the infimum above. Using the results of $\S 5$, we will present a proof of the following result of [Lim]:

Theorem A.1 Any graph $G$ as above carries a unique optimal metric up to scale. In fact, the metric given by

$$
m(e)=\log \sqrt{(d(p)-1)(d(q)-1)}
$$


on the edges joining $p$ and $q$ is optimal, and hence

$$
H(G)=\frac{1}{2} \sum_{p \in V(G)} d(p) \log (d(p)-1) .
$$

Corollary A.2 An optimal metric assigns equal lengths to the edges of $G$ iff $G$ is regular or biregular.

Corollary A.3 We have $h(G, m) \geq(3 n-3) \log 2$ for all unit volume graphs with $\pi_{1}(G) \cong F_{n}$. Equality holds iff $(G, m)$ is a regular graph of degree 3 with all edges of equal length.

Proof. One can check that $a^{a+1} b^{b+1} \leq(a+b-1)^{a+b}$ for all integers $a, b \geq 2$. Then formula (A.1) shows $H(G)$ decreases whenever a vertex with $d(p)>3$ is split into two vertices of smaller degree, while keeping the rank constant. Repeated splitting leads to a regular graph of degree 3.

See also $[\mathrm{KN}]$ for Corollary A.3.

Gradient of entropy. Our treatment of Theorem A.1 will use the language of pressure and the equilibrium measure (defined below), to indicate the connection with the thermodynamic formalism (see e.g. $[\mathrm{PP}]$ ). We will first introduce a directed graph $T G$ which plays the role of a combinatorial tangent bundle for $G$, and then show:

Proposition A.4 The entropy $h(G, m)$ is a real-analytic, strictly convex function on the space of metrics. Its gradient satisfies

$$
\frac{\nabla h}{h}=-\frac{\mu}{\langle\mu, m\rangle},
$$

where $\mu$ is the equilibrium measure for $(G, m)$.

Corollary A.5 A metric is optimal iff its equilibrium measure is constant.

Proof. By Lagrange multipliers, we have $\nabla h=\lambda \nabla(\mathrm{vol})$ at a minimum of $h$ on a level set of $\operatorname{vol}(G, m)$. 
Theorem A.1 follows easily from the Corollary above.

Remarks. Before proceeding to the details, we make four remarks.

1. Constant curvature. It is known that a metric of constant negative curvature is optimal for the volume entropy on a compact manifold, given by the growth rate

$$
h(M, g)=\lim _{T \rightarrow \infty} \log \operatorname{vol}(B(x, T))^{1 / T}
$$

of balls in its universal cover [BCG1]; see also the surveys [BCG2] and [CoF].

The optimal metric given in Theorem A.1 can similarly be viewed as a metric of constant negative curvature on $G$. To see this, note that the universal cover $\widetilde{G}$ is a tree that can be assembled from copies of the balls $B\left(p, r_{\underline{p}}\right), p \in V(G)$, where $r_{p}=(1 / 2) \log (d(p)-1)$. Choose a root vertex $x_{0} \in \widetilde{G}$, and think of $y(x)=d\left(x, x_{0}\right)$ as a height function on the tree. Then a lift of $B\left(p, r_{p}\right)$ gives a subtree of height $\Delta y=\log (d(p)-1)$, that expands from a single branch to $(d(p)-1)$ parallel branches as one moves away from the root (see below).

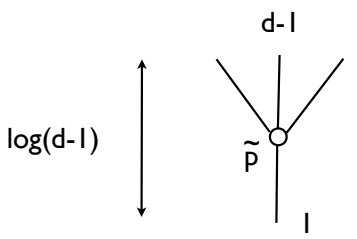

The expansion factor $(d(p)-1)=\exp (\Delta y)$ depends only on the change in height, and agrees with the expansion factor for parallel horocycles in the hyperbolic plane.

2. Convexity and minima. The space of metrics on $G$ with fixed volume is not compact, so despite convexity, the existence of an optimal metric is not automatic. In principle, a sequence of metrics of fixed volume with $h\left(G, m_{n}\right) \rightarrow H(G)$ might limit on a weak metric $m$ which vanishes on some edges of $G$.

This cannot happen if the zero set $Z(m)$ contains a cycle, for in this case $h\left(G, m_{n}\right) \rightarrow \infty$; but what if $Z(m)$ is a union of trees? This point is finessed in the proof of Theorem A.1 by giving an explicit formula for the optimal metric. For a more conceptual argument, one can use Proposition A. 4 to show that $-\nabla h$ points inward at the boundary of the space of positive metrics.

3. Outer space. The outer automorphism group of the free group $F_{n}$ acts on a contractible space $X_{n}$, introduced by Culler and Vogtmann, called outer 
space $[\mathrm{CV}]$. A point of $X_{n}$ is specified by a unit volume graph $(G, m)$ as above, together with a marking $\alpha: F_{n} \cong \pi_{1}\left(X_{n}\right)$. The different topological types of marked graphs give a natural partition of $X_{n}$ into simplicial cells, each swept out by the space of unit volume metrics on a given graph.

It is easy to see that the entropy defines a continuous, $\operatorname{Out}\left(F_{n}\right)$-invariant function on $X_{n}$, which descends to a proper map

$$
h: X_{n} / \operatorname{Out}\left(F_{n}\right) \rightarrow \mathbb{R}_{+} .
$$

Theorem A.1 shows that $h$ has a unique critical point on each open cell of $X_{n}$. Thus one can think of $h$ as a piecewise-smooth Morse function, and the cells of $X_{n}$ as the stable manifolds of $-\nabla h$. The flow generated by $-\nabla h$ provides a retraction of $X_{n}$ to a spine with compact quotient, which may be of independent interest.

The global minima of $h$ are identified by Corollary A.3. For an alternative proof of this Corollary, one can use the fact that the cells of $X_{n}$ corresponding to trivalent graphs are dense.

4. Variant: Entropy of random walks. Instead of closed geodesics we can consider combinatorial paths in $G$, which are allowed to fold back and immediately retrace an edge. Let $N(T)$ denote the number of paths of length $\leq T$, let

$$
\widetilde{h}(G, m)=\lim _{T \rightarrow \infty} \log N(T)^{1 / T},
$$

and let $\widetilde{H}(G)=\inf _{m} \operatorname{vol}(G, m) \widetilde{h}(G, m)$. These variants of the entropy are related to random walks and potential theory on the universal cover of $G$.

The proof of Theorem A.1 can easily be modified to show that

$$
\widetilde{H}(G)=\frac{1}{2} \sum_{V(G)} d(g) \log d(g),
$$

that

$$
m(e)=\log \sqrt{d(p) d(q)}
$$

is an optimal metric for $\widetilde{h}(G, m)$, and that the optimal metric is unique up to scale. A version of Corollary A.3 holds as well, with the bound $\widetilde{h}(G, m) \geq$ $(3 n-3) \log 3$ and the same cases of equality.

The existence of an optimal metric is simpler to prove in this case, because $\widetilde{h}(G, m) \rightarrow \infty$ if $m(e) \rightarrow 0$. This behavior is closer to the case of the entropy function on a fibered face of the Thurston norm ball of a hyperbolic 3-manifold [Mc, §5], which also tends to infinity at the boundary. 
Setting for the proof. We now turn to the proof of Proposition A.4. Let $G$ be a connected graph with $d(p) \geq 3$ for all $p \in V(G)$. Each edge of $G$ has two possible orientations. Let $a, b$ denote edges of $G$ with chosen orientations, and let $a^{\prime}$ denote $a$ with its orientation reversed. We write $a \triangleleft b$ if $b$ starts at the same vertex where $a$ ends, and $a \neq b^{\prime}$.

A geodesic is a sequence of oriented edges $\left(a_{1}, \ldots, a_{n}\right)$ with $a_{i} \triangleleft a_{i+1}$. If $a_{n} \triangleleft a_{1}$ as well, then this data determines an oriented, closed geodesic $C$ in $G$. Conversely, a closed geodesic $C$ determines an edge sequence up to cyclic reordering.

We have a natural directed graph $T G$ that plays the role of a combinatorial tangent bundle for $G$. Its vertex set $V(T G)$ consists of the edges of $G$ with chosen orientations, and $(a, b) \in E(T G)$ iff $a \triangleleft b$. The natural two-toone projection $V(T G) \rightarrow E(G)$ sends directed paths in $T G$ to geodesics in $G$.

Let $C(T G)$ denote the algebra of functions $v: V(T G) \rightarrow \mathbb{R}$, with the inner product

$$
\left\langle v_{1}, v_{2}\right\rangle=\sum_{a} v_{1}(a) v_{2}(a)
$$

We use $v_{1} v_{2}$ and $v_{1}+v_{2}$ to denote pointwise multiplication and addition. Let $A$ and $\tau$ denote the operators on $C(T G)$ defined by

$$
(A v)(a)=\sum_{a \triangleleft b} v(b) \text { and }(\tau v)(a)=v\left(a^{\prime}\right)
$$

they satisfy $\tau^{2}=\mathrm{id}$ and $\tau A \tau=A^{t}$. Note that $A$ simply gives the adjacency matrix of $T G$. We use the shorthand $v^{\prime}=\tau v$, so $v^{\prime}(a)=v\left(a^{\prime}\right)$.

Metrics. A metric on $G$ is given by a function $m \in C(T G)$ with $m^{\prime}=m$ and $m(a)>0$ for all $a$. Its volume is given by $\operatorname{vol}(G, m)=(1 / 2)\langle 1, m\rangle$, since each edge of $G$ appears twice in $T G$. The length of a closed geodesic $C=\left(a_{1}, \ldots, a_{n}\right)$ is given by $L(C, m)=\sum_{1}^{n} m\left(a_{i}\right)$. The length of a general 1-cycle can be defined similarly, and it is easy to see:

Lemma A.6 The space of metrics on $G$ maps injectively into $H^{1}(T G, \mathbb{R})$.

Proof. Let $a$ be an oriented edge of $G$, connecting the vertex $v_{1}$ to $v_{2}$. Our assumptions on $G$ imply there are closed paths $C_{1}$ and $C_{2}$ in $G$ based at $v_{1}$ and $v_{2}$ that avoid $a$. Then $C=C_{1} a C_{2} a^{\prime}$ gives a closed geodesic with $L(C, m)=2 m(a)+L\left(C_{1}, m\right)+L\left(C_{2}, m\right)$. Thus the value of $m(a)$ can be recovered from cohomology class determined by $m$. 
Pressure. Given $\phi \in C(T G)$, the transfer operator $T_{\phi}: C(T G) \rightarrow C(T G)$ is defined by

$$
T_{\phi} v=e^{\phi}(A v) .
$$

Our assumptions on $G$ imply that $T_{\phi}$ is given by a Perron-Frobenius matrix, with leading eigenvalue equal to its spectral radius $\rho=\rho\left(e^{\phi} A\right)$. Thus we have positive right and left eigenvectors $u, v \in C(T G)$ satisfying

$$
u T_{\phi}=\rho u \text { and } T_{\phi} v=\rho v,
$$

and normalized so that $\langle u, v\rangle=1$. With this normalization, the product $\mu=u v \in C(T G)$ depends only on $T_{\phi}$; it represents the equilibrium measure for $\phi$. Note that $\mu$ is a probability measure, in the sense that $\langle\mu, 1\rangle=1$.

The pressure of $\phi$ is defined by $P(\phi)=\log \rho\left(T_{\phi}\right)$. It is a smooth function on the inner product space $C(T G)$, satisfying

$$
\nabla P=\mu \text {. }
$$

Indeed, since the leading eigenvalue of $T_{\phi}$ is simple, we can regard $\rho$ and $v$ (suitably normalized) as smooth functions of $\phi$. Taking the first variation of (A.3) then gives

$$
\left(T_{\phi} v\right)^{\cdot}=\dot{\phi} T_{\phi} v+T_{\phi} \dot{v}=\dot{\rho} v+\rho \dot{v} .
$$

Taking the inner product with $u$ and using (A.3) and the normalization $\langle u, v\rangle=1$, we find

$$
\rho\langle u, \dot{\phi} v\rangle+\rho\langle u, \dot{v}\rangle=\dot{\rho}+\rho\langle u, \dot{v}\rangle,
$$

which implies

$$
\langle\nabla P, \dot{\phi}\rangle=\dot{\rho} / \rho=\langle u, v \dot{\phi}\rangle=\langle\mu, \dot{\phi}\rangle .
$$

Compare [PP, Prop. 4.10].

Symmetric potentials. If $\phi^{\prime}=\phi$, then the equilibrium measure can be expressed in terms of the single eigenvector $v$; in fact, we have

$$
\mu=e^{-\phi} v v^{\prime}
$$

provide we scale $v$ so $\langle\mu, 1\rangle=1$. To verify (A.5) we just need to check that $u=e^{-\phi} v^{\prime}$ is a right eigenvector for $T_{\phi}^{t}$. But the assumption $\phi=\phi^{\prime}$ implies $e^{\phi} \tau=\tau e^{\phi}$, and hence

$$
T_{\phi}^{t} u=A^{t} e^{\phi} e^{-\phi} \tau v=\tau A v=e^{-\phi} \tau e^{\phi} A v=\rho e^{-\phi} \tau v=\rho u .
$$


Equilibrium measure of a metric. Now let $m$ be a metric on $G$, considered as a function $m: V(T G) \rightarrow \mathbb{R}_{+}$satisfying $m=m^{\prime}$. Define a metric $M: E(T G) \rightarrow \mathbb{R}_{+}$by $M((a, b))=m(a)$. Then it is easy to see that $h(T G, M)=h(G, m)$; i.e. the growth rate of closed paths in the tangent space is the same as the growth rate of closed geodesics in the base. Moreover, the weighted adjacency matrix of $(T G, M)$ satisfies

$$
A\left(t^{M}\right)=T_{\phi}
$$

for $\phi=(\log t) m$. By Theorem 3.2, $t=\exp (-h(T G, M))$ is the unique positive solution to the equation $\rho\left(A\left(t^{M}\right)\right)=1$; equivalently, $h(G, m)$ is characterized by the condition

$$
P(-h(G, m) m)=0 .
$$

For brevity, we refer to the equilibrium measure $\mu$ for the symmetric potential $\phi=-h(G, m) m$ as the equilibrium measure for $(G, m)$.

Proof of Proposition A.4. Our assumptions on $G$ imply that $T G$ is strongly connected; thus Theorem 5.2 implies $h(T G, M)$ is a strictly convex and real-analytic function of the cohomology class determined by $M$; and hence by Lemma A.6, $h(G, m)$ is a real-analytic function of $m$. We can also consider $h(G, m)$ as a function of $m$ determined implicitly equation (A.6); then equation (A.4) yields

$$
0=\langle\nabla P, \dot{h} m+h \dot{m}\rangle=\dot{h}\langle\mu, m\rangle+h\langle\mu, \dot{m}\rangle ;
$$

solving for $\dot{h} / h$ gives equation (A.2).

Proof of Theorem A.1. Define $f: V(G) \rightarrow \mathbb{R}$ by $f(p)=(d(p)-1)^{1 / 2}$. On a directed edge running from $p$ to $q$, let $m(a)=\log (f(p) f(q))$, let $\phi(a)=$ $-\log m(a)$, and let $v(a)=f(p)^{-1}$.

We claim $P(\phi)=0$ and $T_{\phi} v=v$. Indeed, we have

$$
\left(T_{\phi} v\right)(a)=e^{\phi(a)} \sum_{a \triangleleft b} v(b)=\frac{1}{f(p) f(q)} \frac{(d(q)-1)}{f(q)}=\frac{1}{f(p)}=v(a) .
$$

Since $\phi=\phi^{\prime}$, the associated equilibrium measure is given by

$$
\mu(a)=e^{-\phi(a)} v(a) v\left(a^{\prime}\right)=f(p) f(q) f(p)^{-1} f(q)^{-1}=1 .
$$

Thus by Corollary A.5, the metric $m(a)$ is optimal, with $h(G, m)=1$. It is unique up to scale by strict convexity of $h(G, m)$, and the value of $\operatorname{vol}(G, m)=H(G)$ is easily computed by rewriting $(1 / 2)\langle m, 1\rangle$ as a sum over the vertices of $G$. 


\section{References}

[AD] J. W. Aaber and N. Dunfield. Closed surface bundles of least volume. Algebr. Geom. Topol. 10(2010), 2315-2342.

[AHR] Y. Algom-Kfir, E. Hironaka, and K. Rafi. Digraphs and cycle polynomials for free-by-cyclic groups. Preprint, 2013.

[BCG1] G. Besson, G. Courtois, and S. Gallot. Entropies et rigidités des espaces localement symétriques de courbure strictement négative. Geom. Funct. Anal. 5(1995), 731-799.

[BCG2] G. Besson, G. Courtois, and S. Gallot. Minimal entropy and Mostow's rigidity theorems. Ergodic Theory Dynam. Systems 16(1996), 623-649.

[Bir] J. S. Birman. PA mapping classes with minimum dilatation and Lanneau-Thiffeault polynomials. Preprint, 2011.

[Bol] B. Bollabás. Graph Theory. Springer, 1979.

[B] D. Boyd. The maximum modulus of an algebraic integer. Math. Comp. 45(1985), 243-249.

[CF] P. Cartier and D. Foata. Problèmes combinatoires de commutation et réarrangements, volume 85 of Lecture Notes in Math. Springer, 1969.

[CH] J. Cho and J. Ham. The minimal dilatation of a genus two surface. Experiment. Math. 17(2008), 257-267.

[CoF $]$ C. Connell and B. Farb. Some recent applications of the barycenter method in geometry. In Topology and geometry of manifolds (Athens, GA, 2001), Proc. Sympos. Pure Math., 71, pages 19-50. Amer. Math. Soc., 2003.

[Csi] P. Csikvári. Note on the smallest root of the independence polynomial. Combin. Probab. Comput. 22(2013), 1-8.

[CV] M. Culler and K. Vogtmann. Moduli of graphs and automorphisms of free groups. Invent. math. 84(1986), 91-119.

[CDS] D. Cvetković, M. Doob, and H. Sachs. Spectra of Graphs. Academic Press, 1980. 
[DKL] S. Dowdall, I. Kapovich, and C. Leininger. McMullen polynomials and Lipschitz flows for free-by-cyclic groups. Preprint, 2013.

[FLM] B. Farb, C. J. Leininger, and D. Margalit. Small dilatation pseudoAnosov homeomorphisms and 3-manifolds. Adv. Math. 228(2011), 1466-1502.

[Fi] D. C. Fisher. The number of words of length $n$ in a graph monoid. Amer. Math. Monthly 96(1989), 610-614.

[Gant] F. R. Gantmacher. The Theory of Matrices, volume II. Chelsea, 1959.

[Gel] A. O. Gel'fond. Transcendental and Algebraic Numbers. Dover, 1960.

[GS] M. Goldwurm and M. Santini. Clique polynomials have a unique root of smallest modulus. Inform. Process. Lett. 75(2000), 127132 .

[HS] J.-Y. Ham and W. T. Song. The minimum dilatation of pseudoAnosov 5-braids. Experiment. Math. 16(2007), 167-179.

[HR] G. H. Hardy and M. Riesz. The General Theory of Dirichlet's Series. Cambridge University Press, 1915.

[Ha] W. J. Harvey. Boundary structure of the modular group. In Riemann surfaces and related topics: Proceedings of the 1978 Stony Brook Conference, pages 245-251. Princeton Univ. Press, 1981.

[Hir] E. Hironaka. Small dilatation mapping classes coming from the simplest hyperbolic braid. Algebr. Geom. Topol. 10(2010), 20412060 .

[KN] I. Kapovich and T. Nagnibeda. The Patterson-Sullivan embedding and minimal volume entropy for outer space. Geom. Funct. Anal. 17(2007), 1201-1236.

[KOR] K. H. Kim, N. S. Ormes, and F. W. Roush. The spectra of nonnegative integer matrices via formal power series. J. Amer. Math. Soc. 13(2000), 773-806.

[KT1] E. Kin and M. Takasawa. Pseudo-Anosov braids with small entropy and the magic 3-manifold. Comm. Anal. Geom. 19(2011), 705-758. 
[KT2] E. Kin and M. Takasawa. Pseudo-Anosovs on closed surfaces having small entropy and the Whitehead sister link exterior. J. Math. Soc. Japan 65(2013), 411-446.

[La] E. Landau. Handbuch der Lehre von der Verteilung der Primzahlen. Teubner, 1909.

[LT] E. Lanneau and J.-L. Thiffeault. On the minimum dilatation of pseudo-Anosov homeomorphisms on surfaces of small genus. Ann. Inst. Fourier 61(2011), 105-144.

[Lim] S. Lim. Minimal volume entropy for graphs. Trans. Amer. Math. Soc. 360(2008), 5089-5100.

[Li] D. Lind. The entropies of topological Markov shifts and a related class of algebraic integers. Ergod. Th. 65 Dynam. Sys. 4(1984), 283-300.

[MM] H. A. Masur and Y. N. Minsky. Geometry of the complex of curves. I. Hyperbolicity. Invent. math. 138(1999), 103-149.

[Mc] C. McMullen. Polynomial invariants for fibered 3-manifolds and Teichmüller geodesics for foliations. Ann. scient. Éc. Norm. Sup. 33(2000), 519-560.

$[\mathrm{Pa}] \quad$ A. Papadopoulos. Geometric intersection functions and Hamiltonian flows on the space of measured foliations on a surface. Pacific J. Math. 124(1986), 375-402.

[PP] W. Parry and M. Pollicott. Zeta Functions and the Periodic Orbit Structure of Hyperbolic Dynamics. Astérisque, vol. 187-188, 1990.

[Pen] R. Penner. Bounds on least dilatations. Proc. Amer. Math. Soc. 113(1991), 443-450.

[PS] M. Pollicott and R. Sharp. A Weil-Petersson type metric on spaces of metric graphs. Geom. Dedicata 172(2014), 229-244.

[Smy] C. J. Smyth. The Mahler measure of algebraic numbers: a survey. In Number Theory and Polynomials, pages 322-349. Cambridge Univ. Press, 2008.

[Sun] H. Sun. A transcendental invariant of pseudo-Anosov maps. Preprint, 2012. 
[Wu] Q. Wu. The smallest Perron numbers. Math. of Comp. 79(2010), 2387-2394. 